ISSN 2073-8994

www.mdpi.com/journal/symmetry

Article

\title{
Dual Symmetry in Bent-Core Liquid Crystals and Unconventional Superconductors
}

\author{
Bruno Mettout ${ }^{1} *$ and Vladimir Lorman ${ }^{2}$ \\ 1 PSC, Faculté des Sciences d'Amiens, Université de Picardie, 33 rue St Leu, 80039 Amiens, France \\ 2 Laboratoire de Physique Théorique et Astroparticules, Université Montpellier II, Place Eugène \\ Bataillon, 34095 Montpellier, France; E-Mail: Vladimir.Lorman@univ-montp2.fr
}

* Author to whom correspondence should be addressed; E-Mail: bruno.mettout@u-picardie.fr

Received: 24 September 2009; in revised form: 17 December 2009 / Accepted: 1 January 2010 / Published: 11 January 2010

\begin{abstract}
We extend the Landau theory of bent-core mesophases and d-wave high- $\mathrm{T}_{\mathrm{c}}$ superconductors by considering additional secondary pseudo-proper order parameters. These systems exhibit a remarkable analogy relating their symmetry groups, lists of phases, and an infinite set of physical tensors. This analogy lies upon an internal dual structure shared by the two theories. We study the dual operator transforming rotations into translations in liquid crystals, and gauge symmetries into rotations in superconductors. It is used to classify the bent-core line defects, and to analyze the electronic gap structure of lamellar d-wave superfluids.
\end{abstract}

Keywords: bent core liquid crystals; unconventional superconductors; dual symmetry

\section{Introduction}

The unconventional class of liquid crystals discovered one decade ago, which is constituted with non-chiral bent-core molecules [1], presents a rich polymorphism [2] with extraordinary features and symmetry properties, the most prominent being the spontaneous onset of chirality in the ordered smectic phases [3]. Despite many attempts to describe these phases with acrobatic combinations of classical order parameters [3-6], smectic density waves, polarization, and tilt vectors, a simpler and more elegant description involving a single vector-wave order parameter $[7,8]$ seems to us necessary to account of the available experimental data [7-9]. In particular, from the thermodynamical point of view 
the fact that a direct transition occurs commonly between the isotropic liquid and the ordered phases is much more likely when a single order parameter is involved. This permits us to predict the structure of the stable phases together with their thermodynamical behaviour [7,8], and more specifically the behaviour of several important physical tensors describing, for instance, the biaxiality, electro-optic response and chirality of the predicted structures. In addition, we have successfully extended our model to almost all observed bi-dimensionally and tri-dimensionally ordered bent-core phases [9].

This single order parameter can be equivalently spanned by either a polarization wave or a tilt wave, since they transform according to the same irreducible representation of the non-chiral euclidean group when the wave vector is different from zero. It combines thus in a single object the degrees of freedom involved by distinct classical order parameters. We have shown in Refs. [7,8] that this model permits to foresee the stabilization of four ordered phases, denoted by $\mathrm{C}, \mathrm{R}$, and EL corresponding, respectively, to linear, circular and elliptic polarizations of the order-parameter wave. $\mathrm{R}$ describes the smectic $C_{P}$ phase predicted in 1992 [10] and then observed in bent-core systems [10,11], EL describes the commonly observed B2 phase [12,13], and we have proposed that $\mathrm{C}$ is an unmodulated approximation of the intriguing B7 phase found in 1999 [2]. In these phases the polarization and tilt waves are "parallel", in a sense explained below. However, we shall show that when one permits the two waves to be "non parallel", i.e., relatively shifted along the smectic normal or relatively rotated, four additional phases can be stabilized. The reason of this unusual extension of the polymorphism, which is characteristic of transitions with continuous-symmetry breakdowns, has been described within the context of superconductivity in Ref. [14].

The bent-core theory exhibits a quite remarkable analogy [7] with the theory of d-wave superconductors in 2D systems [15], such as high- $\mathrm{T}_{\mathrm{c}}$ lamellar superconductors or unconventional superfluid films. This analogy relies upon the fact that the two theories have the same "image group" [16], that is, the same set of matrices associated with the symmetries of the parent, isotropic or normal, phases. The main consequence of this peculiarity is that both systems have the same list of phases with analog symmetry groups, the same theoretical phase diagrams, and the same thermodynamic variations of the primary and secondary order parameters. At first sight the analogy is rather formal since a common matrix can be associated with distinct actual symmetry elements in each system. For instance, a gauge symmetry in superconductors yields the same matrix as a translation in liquid crystals. Thus, we do not expect a common behavior of the same physical quantities. However, the analogy is more subtle for it relates in fact the behaviors of distinct, but analog, tensors. For instance, the macroscopic polarization normal to the smectic planes exhibits exactly the same thermodynamic behavior as the linear magneto-electric suceptibility in superconductors. More precisely, both cancel in analog sets of ordered phases, with the same critical exponents.

This universality property relating distinct systems is a well-known feature of the Landau theory of phase transitions [16-18]. When the image group is small, the physical consequences of the analogy are poor, and the number of analog systems is large, and conversely when the image group is large. For instance, deGennes used such an analogy between s-pairing superconductors and smectic A liquid crystals [19], on the one hand, and between neutral superfluids and smectic C [20], on the other hand. These two analogies bear much information because the image groups are continuous. In the first analogy gauge symmetries correspond to translations in Smectic A, while in the second analogy they correspond to rotations in Smectic C. In both cases the order parameter is bidimensional, describing 
density waves, on the one hand, and the molecular tilt, on the other hand. deGennes extended further the former analogy by proposing a liquid crystal analog of the superconducting gauge-type coupling with the magnetic field [21-23], which goes much beyond the coincidence between image groups.

In the case of bent-core mesophases the image group contains four connected bicontinuous parts $[7,8]$. This very large group yields thus a still more fruitful analogy, which permits to relate precisely the rich polymorphisms of the two systems, and an infinite set of tensors accounting for most of their physical properties. It mixes the two deGennes analogies since both rotations and translations are simultaneously broken in the bent-core ordered mesophases. We shall use this analogy to complete the d-wave theory, and to predict the behavior of its stable phases. In particular, we shall focus attention on the properties of the electronic excitation spectrum, which is a key physical feature of these systems crucially depending on symmetry considerations.

The analogy is complicated by the dual internal structure of the two theories. Indeed, the image groups are products of two isomorphic one-dimensional subgroups, which can be interchanged without modifying the formal structure of the theories. This duality relates rotations and translation in the liquid crystal system, and rotations and gauge symmetries in superconductors. In both cases it defines an inner automorphism of the bicontinuous group, which is realized by a dual operator permuting its subgroups. The fact that the free energies are invariant under this operator yields many interesting consequences. Firstly, it permits to classify all the objects involved in these theories, phases, symmetry groups, and physical tensors, into either self-dual objects or dual-conjugated pairs, revealing many non-trivial features of these systems. Secondly, it provides a deep insight into both mathematical and physical aspects of the theories. We shall show in particular how the classification of line defects in bent-core phases results from the dual character of the order parameter.

\section{The Vector-Wave Model}

The primary order parameter of the vector-wave model describing ordered bent-core mesophases $[7,8]$ is spanned by a transverse polarization wave $\overrightarrow{\mathrm{P}}(\mathrm{z})$ :

$$
\vec{P}(z)=p_{x} \cos \left(k z+\varphi_{x}\right) \vec{e}_{x}+p_{y} \cos \left(k z+\varphi_{y}\right) \vec{e}_{y}
$$

where the axis $\mathrm{Oz}$ is parallel to the wave vector $\vec{k}$, so that its two components remain parallel to Ox and $\mathrm{Oy} . \mathrm{p}_{\mathrm{x}}, \mathrm{p}_{\mathrm{y}}, \varphi_{x}$ and $\varphi_{y}$ are the real amplitudes and phases of the wave. The characteristic features of $\overrightarrow{\mathrm{P}}(\mathrm{z})$ may be more conveniently expressed in terms of the following complex amplitudes :

$$
\eta_{1}=\rho_{1} e^{i \varphi_{1}}=p_{x} e^{i \varphi_{x}}-i p_{y} e^{i \varphi_{y}} \quad, \quad \eta_{2}=\rho_{2} e^{i \varphi_{2}}=p_{x} e^{-i \varphi_{x}}-i p_{y} e^{-i \varphi_{y}}
$$

Although this order parameter contains only four components, $\eta_{1}, \eta_{2}, \eta_{1}^{*}, \eta_{2}{ }^{*}$, the vector wave belongs in fact to an infinite-dimensional irreducible representation of the Euclidean group $\mathrm{O}(3) \times \mathrm{T}_{3}$, where $\mathrm{O}(3)$ is the orthogonal group generated by rotations and inversion, and $\mathrm{T}_{3}$ is the $3 \mathrm{D}$ continuous translation group. It is spanned by an infinite set of waves propagating along all the directions of space. However, since for stabilizing ordered smectic-type phase one needs to consider only the two parallel wave vectors $\vec{k}=\mathrm{k} \vec{e}_{z}$ and $-\vec{k}$ appearing in Eq. (1), one can restrict the symmetry analysis to the 
subgroup $D_{\infty h} \times T_{3}$ of $\mathrm{O}(3) \times \mathrm{T}_{3}$ leaving the set $\{\vec{k}$ and $-\vec{k}\}$ invariant. $D_{\infty h} \times T_{3}$ is generated by the rotations $C_{\varphi}$ around $\mathrm{z}$, the space inversion $\mathrm{I}$, one mirror plane $\sigma_{x}$ parallel to $\vec{k}$ and the translations $\mathrm{T}_{\mathrm{t}}$ parallel to Oz. The action of these symmetries on the complex amplitudes $\left(\eta_{1}, \eta_{2}, \eta_{1}{ }^{*}, \eta_{2}{ }^{*}\right)$ is given by the matrices:

$$
\begin{aligned}
& U_{2 x}=\left(\begin{array}{llll} 
& & & 1 \\
& & 1 & \\
& 1 & & \\
1 & & &
\end{array}\right) \quad \sigma_{x}=\left(\begin{array}{llll} 
& & -1 & \\
& & & -1 \\
-1 & & & \\
& -1 & &
\end{array}\right) \\
& C_{\varphi}=\left(\begin{array}{llll}
e^{-i \varphi} & & & \\
& e^{-i \varphi} & & \\
& & e^{i \varphi} & \\
& & & e^{i \varphi}
\end{array}\right) \quad T_{t}=\left(\begin{array}{llll}
e^{-i k t} & & \\
& e^{i k t} & & \\
& & e^{-i k t} & \\
& & & e^{i k t}
\end{array}\right)
\end{aligned}
$$

One can refine the description of the symmetry-breaking mechanism by considering a secondary wave $\vec{A}(\mathrm{z})$ representing the mean tilt vector of the molecules located at position $\mathrm{z}$ :

$$
\vec{A}(z)=a_{x} \cos \left(k z+\psi_{x}\right) \tilde{e}_{x}+a_{y} \cos \left(k z+\psi_{y}\right) \tilde{e}_{y}
$$

where $\tilde{e}_{x}$ and $\tilde{e}_{y}$ form a basis of unit orthogonal axial vectors. The complex amplitudes of $\overrightarrow{\mathrm{A}}$ are defined as in Eq. (2) :

$$
\xi_{1}=a_{1} e^{i \psi_{1}}=a_{x} e^{i \psi_{x}}-i a_{y} e^{i \psi_{y}} \quad, \quad \xi_{2}=a_{2} e^{i \psi_{2}}=a_{x} e^{-i \psi_{x}}-i a_{y} e^{-i \psi_{y}}
$$

The corresponding matrices of $D_{\infty h} \times T_{3}$ are deduced from those in Eq. (3) by changing the signs of the inversion I and of the mirror plane $\sigma_{\mathrm{x}}$.

The presence of two continuous symmetries in (3) implies that the order parameters split into two "Goldstone angles" $\varphi_{R}$ and $\varphi_{T}$ :

$$
\begin{aligned}
& \varphi_{R}=\frac{\varphi_{R}^{P}+\varphi_{R}^{A}}{2}=\frac{\varphi_{1}+\varphi_{2}}{4}+\frac{\psi_{1}+\psi_{2}}{4} \\
& \varphi_{T}=\frac{\varphi_{T}^{P}+\varphi_{T}^{A}}{2}=\frac{\varphi_{1}-\varphi_{2}}{4}+\frac{\psi_{1}-\psi_{2}}{4}
\end{aligned}
$$

and six "energetic components": On the one hand, $\mathrm{a}_{1}, \mathrm{a}_{2}$ and $\rho_{1}, \rho_{2}$, which describe the shape of $\overrightarrow{\mathrm{A}}$ and $\overrightarrow{\mathrm{P}}$ separately, and, on the other hand, $\varphi_{R}^{P}-\varphi_{R}^{A}, \varphi_{T}^{P}-\varphi_{T}^{A}$ which describe the relative orientation and z-position of the two waves. $\varphi_{R}$ is naturally associated with the subgroup generated by rotations and the space inversion I since it transforms as:

$$
\begin{aligned}
& C_{\phi} \varphi_{R}=\varphi_{R}-\phi \text { and } T_{t} \varphi_{R}=\varphi_{R} \\
& \sigma_{x} \varphi_{R}=-\varphi_{R} \text { and } I \varphi_{R}=\varphi_{R}
\end{aligned}
$$

whereas $\varphi_{T}$ is associated with translations and the mirror plane $\sigma_{\mathrm{x}}$ since: 


$$
\begin{aligned}
& C_{\phi} \varphi_{T}=\varphi_{T} \text { and } T_{t} \varphi_{T}=\varphi_{T}-k t \\
& I \varphi_{T}=-\varphi_{T} \text { and } \sigma_{x} \varphi_{T}=\varphi_{T}
\end{aligned}
$$

In a single domain of any ordered phase $\varphi_{R}$ and $\varphi_{T}$ can be set to zero by suitably choosing the coordinate frame. In the sequel we shall often implicitly assume such a choice.

Eight phases are stabilized. In a first set of four phases already described in Refs. [5] the two order parameters are "parallel" (in a broad sense indicating that the symmetry groups of $\overrightarrow{\mathrm{A}}(\mathrm{z})$ and $\overrightarrow{\mathrm{P}}(\mathrm{z})$ coincide). In this set all the phases, denoted by L, R, C and EL, satisfy the "parallelism" constraints: $\varphi_{R}^{P}=\varphi_{R}^{A}+\frac{\pi}{2}, \varphi_{T}^{P}=\varphi_{T}^{A}+\frac{\pi}{2}(\bmod . \pi)$.

$\underline{\mathrm{L}}$ : Isotropic liquid.

$\underline{\mathrm{R}}$ : Linear phase. $\overrightarrow{\mathrm{A}}$ and $\overrightarrow{\mathrm{P}}$ are both linearly polarized. The plane of polarization of $\overrightarrow{\mathrm{A}}$ is normal to that of $\overrightarrow{\mathrm{P}}$ and the maximums of the primary wave coincide with the nodes (zeroes) of the secondary wave, and reciprocally. Its symmetry is the non-chiral orthorhombic space group Pmma.

$\underline{\mathrm{C}}$ : Circular phase. The two waves are circularly polarized, and $\overrightarrow{\mathrm{A}}$ is everywhere parallel to $\overrightarrow{\mathrm{P}}$. Its chiral helical symmetry is $\infty_{1} 22$ (generated by $C_{\phi} T_{\phi / k}$ and $\mathrm{U}_{2 \mathrm{x}}$ ).

EL : Elliptic phase: The two waves are elliptically polarized. The system of elliptic axes of $\overrightarrow{\mathrm{P}}$ and $\overrightarrow{\mathrm{A}}$ are parallel so that the two fields are parallel at positions where they are either maximum or minimum. The group is $\mathrm{P} 2{ }_{1} 22$.

In the second set of four phases, which were not considered in [7,8], the polar and axial waves are no longer "parallel":

C': The two waves are circularly polarized. $\overrightarrow{\mathrm{A}}(\mathrm{z})$ is shifted along $\mathrm{z}$ with respect to $\overrightarrow{\mathrm{P}}(\mathrm{z})$ so that they are turned everywhere to the same angle $\psi_{2}-\varphi_{2}$. The group is $\infty_{1}$.

$\underline{\mathrm{R}^{\prime}}$ : The two waves are still linearly polarized but the two corresponding planes are no longer perpendicular. The plane of $\overrightarrow{\mathrm{A}}$ is rotated with respect to the plane of $\overrightarrow{\mathrm{P}}$ by an angle $\varphi_{T}^{P}-\varphi_{T}^{A}$. The relationships between the maxima and the zeroes of the two waves in R persist in $\mathrm{R}$ '. The group is $\mathrm{P} 2{ }_{1} / \mathrm{m}$.

$\underline{\mathrm{R}}$ ": The converse situation occurs in $\mathrm{R}$ ". The two polarization planes are again perpendicular, but the maximums of one wave are now shifted along $\mathrm{z}$ with respect to the zeroes of the other wave to a distance $2 k \Delta z=\varphi_{R}^{P}-\varphi_{R}^{A}-\frac{\pi}{2}$. The group is $\mathrm{P} 2{ }_{1}$ ma.

$\underline{E L}$ : The two waves are elliptically polarized and oriented along independent directions. The group is $\mathrm{P} 2$.

We present in Fig. 1 schemes of the molecular structures associated with the various ordered phases and the group-subgroup relationships characterizing this polymorphism. We have discussed in our previous articles the assignment of the observed phases with our predictions for unprimed phases and 2D and 3D structures stabilized when one considers several waves with non-parallel wave vectors. 
Figure 1. (a) Group-subgroup relationships between the ordered phases. (b) Scheme of the molecular organization in the various stable phases of the vector-wave model. The difference between $R$ and $R^{\prime}$ is rather subtle: (i) The direction of the polarization in $R^{\prime}$ is not fixed by symmetry, so that the molecular plane can rotate when the temperature is changed. (ii) The molecular fluctuations are less symmetric in R', which cannot appear in the figure since we represent only the mean orientation at the positions of maximum density.

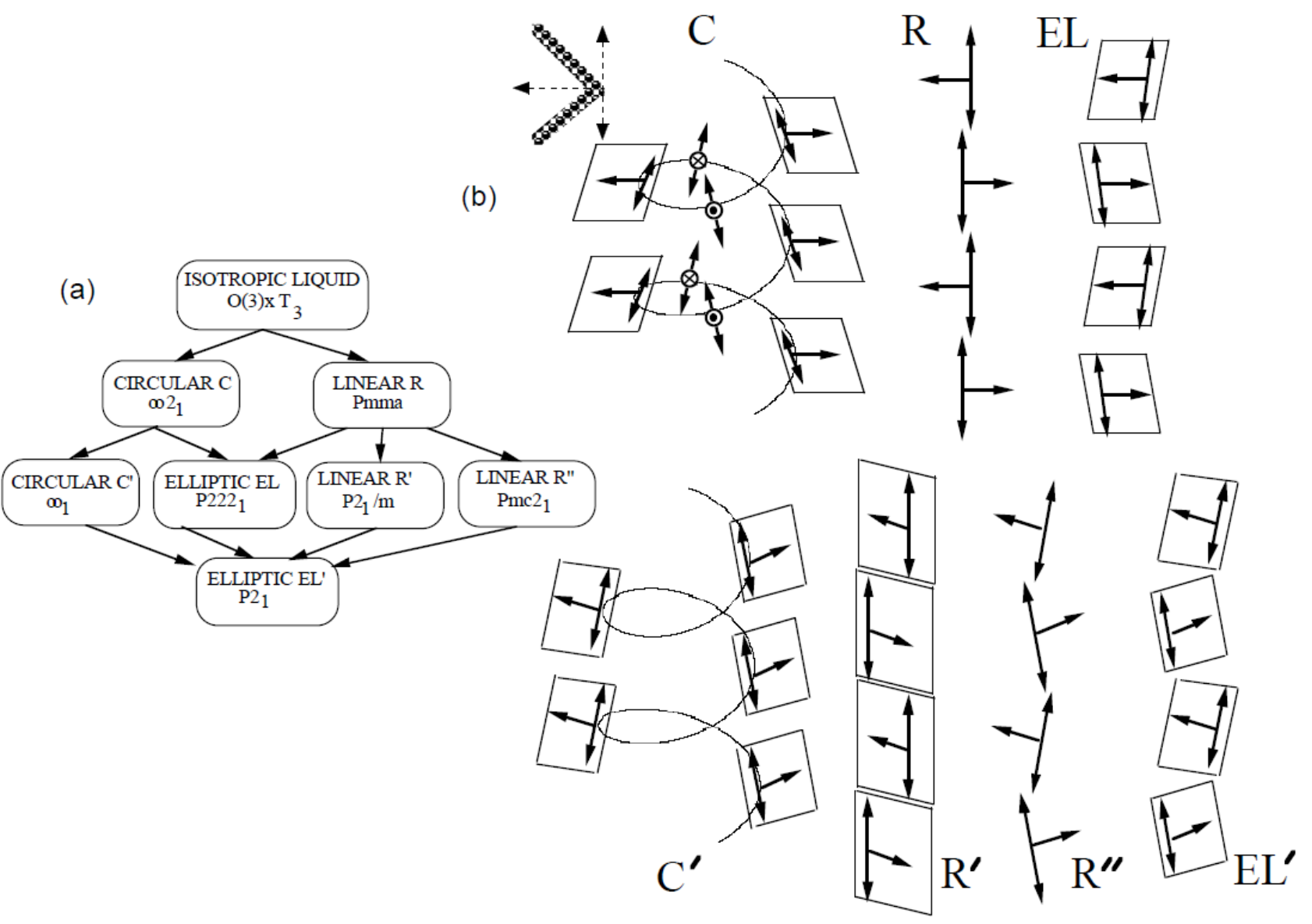

Let us notice that our thermodynamical analysis is based on the homogeneous part of the free energy which does not contain the gradient invariants of the Ginzburg-Landau approach. The model yields only homogeneous stable states. For the inhomogeneous states it gives the access only to their local structure, but not to the global one. We have shown in Ref. [7,8] that homogeneous single domains of the phases $\mathrm{C}$ and $\mathrm{R}$ can be stabilized, while EL, because of its spontaneous chirality, is automatically submitted to an incommensurate longitudinal deformation. We expect also that this phase presents transverse deformations, which could account for the complex inhomogeneous spatial structure reported experimentally in the bent-core phase B7.

Numerous phases and subphases have yet been observed in bent-core systems. They are usually identified by characteristic textures, while their molecular organization and space groups are often not known exactly. In particular variants associated with a single texture can be associated, or not, with distinct symmetries, i.e. distinct phases. We have identified sixteen among the main phases and 
subphases within the vector-wave model. On the one hand, most of them are two- or threedimensional, so that waves with non-parallel wave vectors participate to the ordered structure. On the other hand, the one-dimensional phases are (provisionally) identified as follow: $\mathrm{SmC}_{\mathrm{P}}=\mathrm{R}, \mathrm{B} 2=\mathrm{EL}, \mathrm{C}^{\prime}$ or $\mathrm{EL}^{\prime}, \mathrm{B} 6=\mathrm{R}^{\prime}$ or $\mathrm{R}^{\prime}, \mathrm{B} 7=\mathrm{C}, \mathrm{C}^{\prime}$ or $\mathrm{EL} \mathrm{L}^{\prime}, \mathrm{Sm0}=\mathrm{EL}$, and $\mathrm{B} 8$ is a subphase of $\mathrm{EL}^{\prime}$ obtained with an additional homogeneous order parameter.

The physical behavior of each phase is characterized by a number of secondary tensors waves. Among the most important one may notice: (i) Density waves, which give the phases R, R', R', EL and EL' the structure of layered smectic phases. (ii) Macroscopic polarization along Oz arising in R" and EL'. (iii) Anisotropic components of the optic tensor in the monoclinic and orthorhombic linear and elliptic phases.

In order to classify the infinite set of tensors waves let us introduce the following convenient notation, in which in most cases two integers, namely [n,p], are sufficient: One [n] indicates its tensor properties, and the other [p] its wave vector. These numbers refer to the irreducible representations of the Euclidean group $\mathrm{O}(3) \times \mathrm{T}_{3}$. For non-zero wave vectors, the tensor waves are classified according to the 2D-rotation little group $\mathrm{SO}(2)$ of $\vec{k}$.

(a) First, let us denote a 2D-tensor of rank $n>0$ with the symbol $\Gamma_{n}$ and, for $n=0$ by its parity \pm with respect to the mirror plane $\sigma_{x}$ (scalar $\Gamma_{0}^{+}$, and pseudo scalar $\Gamma_{0}$ ). For instance, a polar vector $\left(\mathrm{V}_{\mathrm{x}}, \mathrm{V}_{\mathrm{y}}, \mathrm{V}_{\mathrm{z}}\right)$, splits into one $2 \mathrm{D}$-vector $\left(\Gamma_{1}: \mathrm{V}_{\mathrm{x}}, \mathrm{V}_{\mathrm{y}}\right)$ plus one even $2 \mathrm{D}$-scalar $\left(\Gamma_{0}^{+}: \mathrm{V}_{\mathrm{z}}\right)$.

(b) Since all the ordered phases are periodic the tensor waves have wave vectors $\vec{K}=\mathrm{p} \vec{k}$ (p integer). At $\mathrm{p}=0$ there is a single homogeneous "wave", which can be either even or odd under I. Hence, the classification of waves is similar to that of $2 \mathrm{D}$-tensors in (a), and one may use the same symbols $\Gamma_{p \neq 0}$, $\Gamma_{0}^{+}$and $\Gamma_{0}$ for representing the corresponding waves.

We shall denote now symbolically by $\Gamma_{n}^{( \pm)} \otimes \Gamma_{p}^{( \pm)}$a tensor wave of rank n and wave vector $\mathrm{p} \vec{k}$. Its dimension is either $1\left(\Gamma_{0}^{ \pm} \otimes \Gamma_{0}^{ \pm}\right), 2\left(\Gamma_{0}^{ \pm} \otimes \Gamma_{\mathrm{p}}\right.$ or $\left.\Gamma_{\mathrm{p}} \otimes \Gamma_{0}^{ \pm}\right)$or $4\left(\Gamma_{\mathrm{n}} \otimes \Gamma_{\mathrm{p}}\right)$. The index $\mathrm{n}$ indicates how the wave transforms under rotations, and $\mathrm{p}$ how it transforms under translations. For instance, there are two polar-vector waves with wave vector $\vec{k}$ : One transverse wave $\left(\Gamma_{1} \otimes \Gamma_{1}\right)$ which coincides with the primary order parameter of our model, and one longitudinal wave $\left(\Gamma_{0}^{+} \otimes \Gamma_{1}\right)$.

We classify these tensor waves with respect to the group $\mathrm{G}_{\mathrm{L}}=D_{\infty h} \times T_{1}$, which is isomorphic to the abstract group $\mathrm{O}(2) \otimes \mathrm{O}(2)$. The first copy of the $2 \mathrm{D}$ orthogonal group $\mathrm{O}(2)$ is $C_{\infty \mathrm{v}}$, generated by the rotations about $\mathrm{Oz}$ and the mirror plane $\sigma_{x}$. The second copy contains the group $\mathrm{T}_{1}$ of translations along $\mathrm{Oz}$ (mod. $2 \pi / \mathrm{k}$ since we consider only waves with wave vectors $\mathrm{p} \vec{k}$ ) and the space inversion $\mathrm{I}$. Thus the image group can be rewritten as:

$$
\mathrm{G}_{\mathrm{L}}=\mathrm{D}_{\infty \mathrm{h}} \times \mathrm{T}_{1}=\left(\mathrm{C}_{\infty} \times \mathrm{C}_{\mathrm{S}}\right) \otimes\left(\mathrm{T}_{1} \times \mathrm{C}_{\mathrm{i}}\right)=\mathrm{O}_{\mathrm{R}}(2) \otimes \mathrm{O}_{\mathrm{T}}(2)
$$

where $\times$ indicates the semi-direct product, and $\otimes$ the direct product. The indices $\mathrm{R}$ and $\mathrm{T}$ denote the rotation and translation $\mathrm{O}(2)$-subgroups of $\mathrm{G}_{\mathrm{L}} \cdot \mathrm{C}_{\mathrm{S}}=\left\{\mathrm{e}, \sigma_{x}\right\}$ and $\mathrm{C}_{\mathrm{i}}=\{\mathrm{e}, \mathrm{I}\}$ (rigorously speaking the actual image group is rather $\mathrm{G}_{\mathrm{L}} /\left\{\mathrm{e}, C_{\pi} T_{\pi / k}\right\}$ since $C_{\pi} T_{\pi / k}$ is represented by the identity matrix in Eq. (3)). 
The previous classification of tensor waves is directly related to the representations of $\mathrm{G}_{\mathrm{L}}$. The irreducible representations of $\mathrm{O}_{\mathrm{T}}(2)$ and $\mathrm{O}_{\mathrm{R}}(2)$ are both labeled $\Gamma_{0}^{+}, \Gamma_{0}^{-}$and $\Gamma_{\mathrm{n}}$ (n positive integer). For $\mathrm{O}_{\mathrm{R}}(2), \Gamma_{0}{ }^{+}$and $\Gamma_{0}^{-}$represent $2 \mathrm{D}$-scalars, respectively even and odd with respect to $\sigma_{x}$, and $\Gamma_{\mathrm{n}}$ represents a $2 \mathrm{D}$-tensor of rank $\mathrm{n}$. In $\mathrm{O}_{\mathrm{T}}(2), \Gamma_{0}{ }^{+}$and $\Gamma_{0}{ }^{-}$represent homogeneous quantities respectively even and odd with respect to I, and $\Gamma_{\mathrm{p}}$ represents a wave with wave vector $\mathrm{p} \vec{k}$. Then the full set of tensor waves can be classified according to the following irreducible representations of $\mathrm{O}_{\mathrm{R}}(2) \otimes \mathrm{O}_{\mathrm{T}}(2)$ :

$\Gamma_{0}^{+} \otimes \Gamma_{0}^{+}$: homogeneous scalar, invariant under $\sigma_{x}$ and I (e.g., density).

$\Gamma_{0}^{+} \otimes \Gamma_{0}^{-}$: homogeneous scalar, invariant under $\sigma_{x}$ and odd under $\mathrm{I}\left(\mathrm{P}_{\mathrm{z}}^{(0)}\right)$.

$\Gamma_{0}^{-} \otimes \Gamma_{0}^{+}$: homogeneous scalar, odd under $\sigma_{x}$ and invariant under I $\left(\mathrm{A}_{\mathrm{z}}^{(0)}\right)$.

$\Gamma_{0}^{-} \otimes \Gamma_{0}^{-}$: homogeneous scalar, odd under $\sigma_{x}$ and I (chiral index $\chi$ ).

$\Gamma_{\mathrm{n}} \otimes \Gamma_{0}^{+}$: homogeneous tensor of rank $\mathrm{n}$, symmetric under $\mathrm{I}\left(\mathrm{A}_{\mathrm{x}}^{(0)}, \mathrm{A}_{\mathrm{y}}^{(0)}\right.$ for $\left.\mathrm{n}=1\right)$.

$\Gamma_{\mathrm{n}} \otimes \Gamma_{0}^{-}$: homogeneous tensor of rank $\mathrm{n}$, odd under $\mathrm{I}\left(\mathrm{P}_{\mathrm{x}}^{(0)}, \mathrm{P}_{\mathrm{y}}^{(0)}\right.$ for $\left.\mathrm{n}=1\right)$.

$\Gamma_{0}^{+} \otimes \Gamma_{\mathrm{p}}$ : scalar wave with wave vector $\mathrm{p} \vec{k}$ (density wave or $\mathrm{P}_{\mathrm{z}}(\mathrm{z})$ for $\mathrm{p}=1$ ).

$\Gamma_{0}^{-} \otimes \Gamma_{\mathrm{p}}$ : pseudo-scalar wave with wave vector $\mathrm{p} \vec{k}$ (e.g., $\mathrm{A}_{\mathrm{z}}(\mathrm{z})$ for $\mathrm{p}=1$ ).

$\Gamma_{\mathrm{n}} \otimes \Gamma_{\mathrm{p}}$ : tensor wave with rank $\mathrm{n}$ and wave vector $\mathrm{p} \vec{k}$ (e.g., order parameters $\overrightarrow{\mathrm{A}}(\mathrm{z})$ and $\overrightarrow{\mathrm{P}}(\mathrm{z})$ for $\mathrm{n}=\mathrm{p}=1)$.

The chiral index $\chi$ is a pseudo scalar, which vanishes in non-chiral phases and changes its sign in two domains of opposite handedness in chiral phases. $\overrightarrow{\mathrm{A}}^{(0)}$ and $\overrightarrow{\mathrm{P}}^{(0)}$ are homogeneous axial and polar vectors, whereas $\overrightarrow{\mathrm{A}}(\mathrm{z})$ and $\overrightarrow{\mathrm{P}}(\mathrm{z})$ are axial and polar transverse vector waves with wave vector $\vec{k}$. The transverse components of the axial vector $\vec{A}^{(0)}$ represent the tilt vector. Its longitudinal component $A_{z}^{(0)}$ can be interpreted as the component $t_{x x z}+t_{y y z}$ of the second-order electroclinic tensor $t_{i j k}$ defined by $\delta \mathrm{A}_{\mathrm{i}}^{(0)}=\mathrm{t}_{\mathrm{ijk}} \mathrm{E}_{\mathrm{j}} \mathrm{E}_{\mathrm{k}}$, which describes the action of high electric fields on the homogeneous tilt vector. The dielectric and optic tensors $\left\{\varepsilon_{x x}-\varepsilon_{y y}, \varepsilon_{x y}\right\}$ are examples of $\Gamma_{2} \otimes \Gamma_{0}^{+}$, whereas the first-order electroclinic tensor $\left\{\varepsilon_{\mathrm{xx}}^{\mathrm{c}}-\varepsilon_{\mathrm{yy}}^{\mathrm{c}}, \varepsilon_{\mathrm{xy}}^{\mathrm{c}}\right\}$, where $\delta \mathrm{A}_{\mathrm{i}}^{(0)}=\varepsilon_{\mathrm{ij}}^{\mathrm{c}} \mathrm{E}_{\mathrm{j}}$, provides an example of $\Gamma_{2} \otimes \Gamma_{0}^{-}$.

These tensors permit also to visualize geometric (translational and rotational) aspects of the wave $\overrightarrow{\mathrm{P}}(\mathrm{z})$. To isolate its translational properties let us define the scalar function :

$$
\mathrm{M}(\mathrm{z})=\mathbf{P}(\mathrm{z}) \cdot \mathbf{P}(\mathrm{z})=\mathrm{n}_{\mathrm{L}}+\mathrm{A}_{\mathrm{T}} \operatorname{Cos}(2 \mathrm{kz})+\mathrm{B}_{\mathrm{T}} \operatorname{Sin}(2 \mathrm{kz})
$$

where $\mathrm{n}_{\mathrm{L}}$ the scalar $\rho_{1}^{2}+\rho_{2}^{2}$, and $\left[A_{T}, B_{T}\right]=2 \rho_{1} \rho_{2}\left[\cos \left(\phi_{1}-\phi_{2}\right),-\sin \left(\phi_{1}-\phi_{2}\right)\right]$ transforms as $\Gamma_{2} \otimes \Gamma_{0}^{+}$. To visualize $\mathrm{M}(\mathrm{z})$ we consider $\mathrm{M}$ and $\mathrm{kz}$ as the radial and angular polar coordinates, respectively, in an abstract plane where the function $\mathrm{M}(\mathrm{z})$ defines then a closed orthorhombic curve (Fig. 2a). It is invariant under a rotation $\mathrm{C}_{\varphi}$, and it rotates under a translation $\mathrm{T}_{\mathrm{t}}$. In the EL-phase the curve has the shape of a double balloon which becomes circular in the C-phase, and which splits into two parts in the R-phase. The splitting results from the cancellation of $\mathbf{P}(z)$ at periodic positions, denoted by "nodes" of the wave, along $\mathrm{z}$ in $\mathrm{R}$. The presence of a node is associated with specific symmetry elements that we shall discuss below. Let us notice that a "dual" description of the angular properties of the wave is provided by the $\Gamma_{2} \otimes \Gamma_{0}^{+}$-type tensor : $\left[A_{R}, B_{R}\right]=2 \rho_{1} \rho_{2}\left[-\cos \left(\phi_{1}+\phi_{2}\right), \sin \left(\phi_{1}+\phi_{2}\right)\right]$. Finally, these two tensors and corresponding figures can be similarly defined with the secondary wave $\overrightarrow{\mathrm{A}}$ (z) in order to distinguish graphically primed and unprimed phases. 
All tensors and tensor waves (except density) vanish in the isotropic liquid and can get finite values in one or several ordered phases. The list of the corresponding "permitted" tensors is given in Table 1 for all the ordered phases.

Figure 2. (a) Graphic representation of the unprimed bent-core phases. The angular coordinate represents the position $\mathrm{z}$ in the unit cell (a complete rotation in the graphic plane is associated with a translation of one unit cell $\lambda$ ), whereas the radial coordinate represents the modulus of the polarization at position $\mathrm{z}$. In the circular $\mathrm{C}$ phase the polarization modulus does not vary with $\mathrm{z}$. In EL the modulus varies periodically yielding a bean-shape curve, but it vanishes nowhere. In $\mathrm{R}$ the small axis of the bean vanishes along directions corresponding to the nodes of the polarization wave. (b) Angular variations of the wave function modulus in the superconducting states. It is isotropic in phase I, orthorhombic without nodes in phase III, and exhibits four nodes in phase II.

(a)

bent-core mesophases

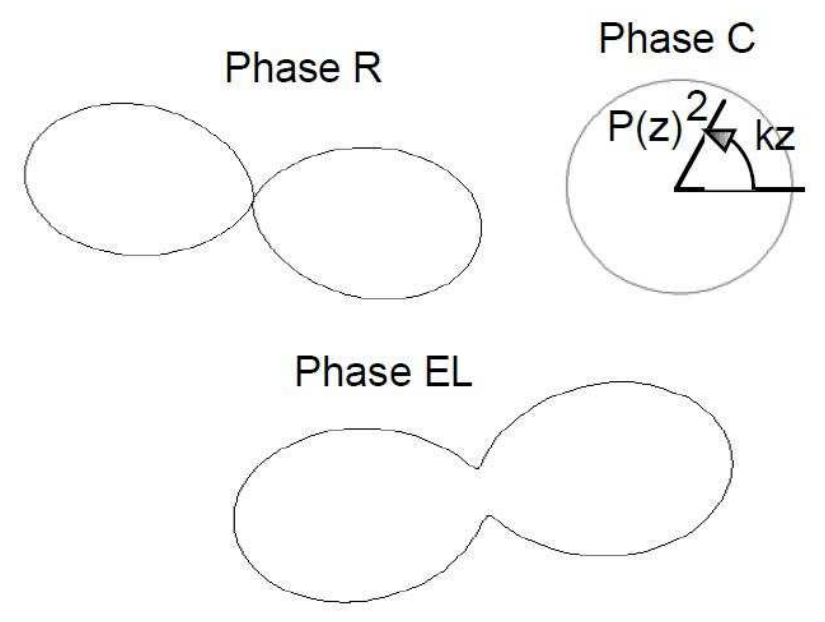

(b) superconducting phases

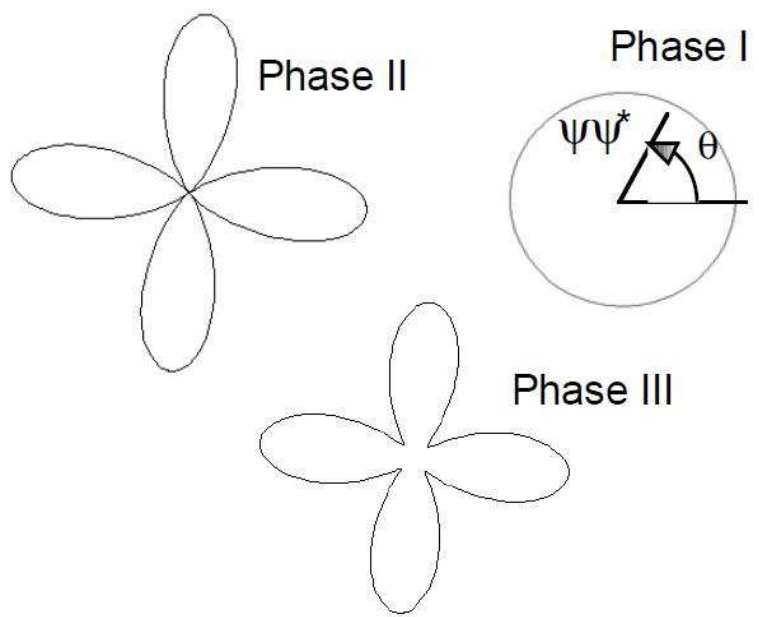

Table 1. First line: Tensor-wave types. Second line: Physical meaning of the waves. The superscript (0) indicates that the tensor is homogeneous. For each tensor wave, the list of phases in which they take non-zero values is indicated in the third line.

\begin{tabular}{|c|c|c|c|c|c|c|}
\hline$\Gamma_{0}^{+} \otimes \square \Gamma_{0}^{-}$ & $\Gamma^{\otimes} \Gamma_{0}^{+}$ & $\Gamma_{0}^{-} \otimes \Gamma_{0}^{-}$ & $\begin{array}{l}\Gamma_{0}^{+} \otimes \Gamma_{\mathrm{p}} \\
\Gamma_{\mathrm{n}} \otimes \Gamma_{0}^{+}\end{array}$ & $\Gamma_{0}{ }^{-} \otimes \Gamma_{\mathrm{p}}$ & $\Gamma_{\mathrm{n}} \otimes \Gamma_{0}^{-}$ & $\Gamma_{\mathrm{n}} \otimes \Gamma_{\mathrm{p}}$ \\
\hline $\begin{array}{l}\text { polarization } \\
\mathrm{P}_{\mathrm{z}}^{(0)}\end{array}$ & tilt $A_{z}^{(0)}$ & $\begin{array}{l}\text { chiral } \\
\text { index } \chi\end{array}$ & $\begin{array}{l}\mathrm{P}_{\mathrm{z}}(\mathrm{z}) \mathrm{n}=1 \\
\mathrm{~A}_{\mathrm{x}}^{(0)}, \mathrm{A}_{\mathrm{y}}^{(0)} \mathrm{p}=1\end{array}$ & $\mathrm{~A}_{\mathrm{z}}(\mathrm{z}) \mathrm{p}=1$ & $\mathrm{P}_{\mathrm{x}}^{(0)}, \mathrm{P}_{\mathrm{y}}^{(0)} \mathrm{n}=1$ & $\begin{array}{l}\overrightarrow{\mathrm{A}} \quad(\mathrm{z}) \quad \text { or } \quad \overrightarrow{\mathrm{P}} \quad(\mathrm{z}) \\
\mathrm{n}=\mathrm{p}=1\end{array}$ \\
\hline $\mathrm{C}^{\prime} \mathrm{R}{ }^{\prime} \mathrm{EL}$ & $\begin{array}{ll}C^{\prime} & \mathrm{R}^{\prime} \\
\mathrm{EL}^{\prime} & \end{array}$ & $\begin{array}{lll}\text { C } & C^{\prime} & \text { EL } \\
\text { EL' } & \end{array}$ & $\begin{array}{l}\text { n even: } \\
\text { R,R',R', } \\
\text { EL,EL' }\end{array}$ & $\frac{\mathrm{n} \text { even }:}{\mathrm{R}^{\prime}, \mathrm{EL}, \mathrm{EL}^{\prime}}$ & $\frac{\mathrm{n} \text { even : }}{\mathrm{R} \text { ',EL,EL'}}$ & $\begin{array}{l}\underline{\mathrm{n}+\mathrm{p} \text { even }:} \\
\mathrm{R}, \mathrm{EL}, \mathrm{EL}^{\prime}, \mathrm{R}^{\prime}, \mathrm{R}^{\prime} \\
\mathrm{n}=\mathrm{p}: \mathrm{C}, \mathrm{C}^{\prime}\end{array}$ \\
\hline
\end{tabular}


One sees that if a phase is symmetric under the screw rotation $C_{\pi} T_{\pi / k}$, then the tensor waves with $n+p$ odd vanish. This rule forbids, for instance, transverse ferrielectricity (because $\mathrm{P}_{\mathrm{x}}^{(0)}=\mathrm{P}_{\mathrm{y}}^{(0)}=0$ ) and ferriclinicity $\left(\mathrm{A}_{\mathrm{x}}^{(0)}=\mathrm{A}_{\mathrm{y}}^{(0)}=0\right)$, on the one hand, and longitudinal antiferroelectricity (cancellation of the wave $\mathrm{P}_{\mathrm{z}}(\mathrm{z})$ with period $\lambda$ ), and anticlinicity (wave $\mathrm{A}_{\mathrm{z}}(\mathrm{z})$ with period $\lambda$ ), on the other hand. Then, the ordered phases can only be transversely antiferro- and longitudinally ferro- electric or -clinic. Likewise, only even harmonics of the density wave can condense, so that the smectic character of the phases corresponds always to a bilayer ordering ( $\operatorname{smectic}$ period $=\lambda / 2$ ).

More generally, Table 1 displays the main physical effects permitting one to distinguish experimentally the various ordered phases arising in the model :

(i) In $\mathrm{C}$, all the tensor waves with $\mathrm{n}=\mathrm{p}$ condense and are circularly polarized. The corresponding polarization has, of course, always the same handedness as the structure. Since phase $\mathrm{C}$ is chiral, $\chi$ takes opposite values in domains with different handedness. $\mathrm{C}$ remains non-smectic (zero density wave), non-polar, and optically uniaxial. In contrast, in $\mathrm{C}^{\prime}$ a homogeneous polarization $\left(\mathrm{P}_{\mathrm{z}}^{(0)}\right)$ takes place along the helix axis. Moreover the presence of a homogeneous longitudinal tilt vector along $\mathrm{Oz}$ $\left(\mathrm{A}_{\mathrm{z}}^{(0)}\right)$ leads to a macroscopic second-order electroclinic effect.

(ii) $\mathrm{R}$ is smectic (with period $\lambda / 2$ ), non-chiral, and optically biaxial $\left(\varepsilon_{x y}\right)$. The $\mathrm{R} \rightarrow \mathrm{R}$ ' transition is characterized by the onset of a second-order electroclinic effect $\left(t_{x x z}+t_{y y z}\right)$. But, in contrast to C', no homogeneous polarization arises along the helix axis. Conversely, at the $\mathrm{R} \rightarrow \mathrm{R}$ " transition the system becomes longitudinally ferroelectric, but without any second-order electroclinic effect.

(iii) At the $\mathrm{C} \rightarrow \mathrm{EL}$ transition first-order electroclinic coefficients $\left(\varepsilon_{\mathrm{ij}}^{\mathrm{c}}\right)$ arise, and the phase becomes optically biaxial $\left(\varepsilon_{i j}\right)$ and smectic. At the $\mathrm{R} \rightarrow \mathrm{EL}$ transition, the system becomes chiral $(\chi)$ and firstorder electroclinic $\left(\varepsilon_{\mathrm{ij}}^{\mathrm{c}}\right)$. At the $\mathrm{EL} \rightarrow \mathrm{EL}$ ' transition the system becomes homogeneously polarized along $\mathrm{Oz}$, and begins to exhibit a second-order electroclinic effect $\left(\mathrm{t}_{\mathrm{xxz}}+\mathrm{t}_{\mathrm{yyz}}\right)$. Finally, at the $\mathrm{R}^{\prime} \rightarrow \mathrm{EL}^{\prime}$ transition the system becomes chiral, polar along $\mathrm{Oz}$ with first-order electroclinicity, whereas at the $\mathrm{R}$ " $\rightarrow$ EL' transition the chirality is accompanied by a longitudinal pseudo-scalar wave with period $\lambda / 2$ and a second-order electroclinic tensor.

Table 1 exhibits spectacular regularities: In all the phases, except R' and R", if a tensor wave of the type $\Gamma_{(\mathrm{R})} \otimes \Gamma_{(\mathrm{T})}\left(\mathrm{R}, \mathrm{T}=\mathrm{n},{ }_{0}^{+}\right.$or $\left.{ }_{0}^{-}\right)$is permitted, then the "dual" tensor $\Gamma_{(\mathrm{T})} \otimes \Gamma_{(\mathrm{R})}$ is also permitted. Furthermore, if $\Gamma_{(\mathrm{R})} \otimes \Gamma_{(\mathrm{T})}$ is permitted in R' then $\Gamma_{(\mathrm{T})} \otimes \Gamma_{(\mathrm{R})}$ is permitted in R'. Finally, all tensors of the symmetric type $\left(\Gamma_{(\mathrm{T})} \otimes \Gamma_{(\mathrm{T})}\right)$ and only them, are permitted in $\mathrm{C}$. The following section is devoted to explain and formalize these regularities that we shall denote by the rotation/translation dual character of the vector-wave model.

\section{The Dual Symmetry}

The properties of the two Goldstone angles $\varphi_{T}$ and $\varphi_{R}$ shown in Eq. 6 together with the previous classification of tensor waves reveal the duality relating translations and rotations in the vector-wave model. This duality originates (i) in the structure of the image group $G_{L}$ (Eq. 7), in which the translation subgroup $\mathrm{T}_{1} \times \mathrm{C}_{\mathrm{i}}$ is isomorphic to the rotation subgroup $C_{\infty \mathrm{v}}$, (ii) in the fact that the tensorwave representation associated with the order parameter $\overrightarrow{\mathrm{P}}(\mathrm{z})$ is $\Gamma_{1} \otimes \Gamma_{1}$, in which the indices have the same unit value $(n=p=1)$ for its tensor (rotations) and wave (translations) aspects. This duality relates different symmetry operations (e.g., rotations $C_{\phi}$ to translations $T_{\phi / k}$ ), different tensor fields (e.g., 
$\Gamma_{1} \otimes \Gamma_{2}$ to $\left.\Gamma_{2} \otimes \Gamma_{1}\right)$ different components of the order parameter $\left(\varphi_{T}\right.$ to $\left.\varphi_{R}\right)$ and different phases (e.g. $\mathrm{R}$ ' to R"). It permits one to explain also various specific properties of "self-dual" objects such as $C_{\phi} T_{\phi / k}, \Gamma_{1} \otimes \Gamma_{1}$ or the C-phase.

The duality idea can be rigorously formalized by means of a "dual operator" D, defined by the following matrix in the primary order-parameter space $\left(\eta_{1}, \eta_{2}, \eta_{2}{ }^{*}, \eta_{1} *\right)$ :

$$
D=\left(\begin{array}{llll}
1 & 0 & 0 & 0 \\
0 & 0 & 1 & 0 \\
0 & 1 & 0 & 0 \\
0 & 0 & 0 & 1
\end{array}\right)
$$

and by the same matrix in the secondary $\left(\xi_{1}, \xi_{2}, \xi_{2} *, \xi_{1} *\right)$ space. The fact that D transforms translation-like objects into rotation-like ones is made clear by using the following notation for the basis functions in the order-parameter spaces : $| \pm \pm\rangle=e^{ \pm i k z} \vec{e}_{ \pm}$, where the first index \pm is associated with the wave part $\left(e^{ \pm i k z}\right)$ of the basis function (appearing in Eqs. (1) and (4)) and the second index with its vector part $\left(\vec{e}_{ \pm}=\vec{e}_{x} \pm i \vec{e}_{y}\right)$. Then, D simply permutes the wave and vector indices: $D|a b\rangle=|b a\rangle$ (note that any combination of $\mathrm{D}$ with a symmetry of $\mathrm{G}_{\mathrm{L}}$ might be used as well as the definition of a possible duality operator). Furthermore, D permutes also the elements of $\mathrm{O}_{\mathrm{R}}(2)$ with those of $\mathrm{O}_{\mathrm{T}}(2)$ :

$$
\mathrm{DC}_{\phi} \mathrm{T}_{\mathrm{t}} \mathrm{D}=\mathrm{C}_{\mathrm{kt}} \mathrm{T}_{\phi / \mathrm{k}}, \mathrm{DID}=\sigma_{\mathrm{x}}
$$

If one combines D with $\mathrm{G}_{\mathrm{L}}$, then one finds an "extended" group $\tilde{G}_{L}$ which contains "dual-free" elements $g$ (belonging to $\mathrm{G}_{\mathrm{L}}$ ) together with dual combinations $\mathrm{gD}$ of $\mathrm{D}$ with dual-free operations. $\tilde{G}_{L}$ is not a symmetry group in the usual sense because $\mathrm{D}$ is a classificatory operation, which has a descriptive role, and not a dynamical symmetry constraining the physical properties of the system. However, the free energy of the vector-wave model is invariant under D, so that the list of lowsymmetry phases and their thermodynamic properties have well-defined dual features (e.g., dual pairs of phases appear in symmetric parts of the theoretical phase diagram). This makes a classification of the phases based on the duality operator consistent. We denote by $\tilde{G}_{p h}$ the extended symmetry group of the phase "ph" (=C, R, EL, R'...), defined as the subgroup of $\tilde{G}_{L}$ leaving $\overrightarrow{\mathrm{P}}(\mathrm{z})$ and $\overrightarrow{\mathrm{A}}(\mathrm{z})$ invariant.

In the domain obtained by canceling the angles $\varphi_{R}$ and $\varphi_{T}$ the extended groups of $\mathrm{C}, \mathrm{R}$, and EL contain D itself. This property is reminiscent of the "magnetic groups" in which the time-reversal operation (formally analogous to D in our approach) may be or not combined with spatial symmetries. When a group contains the time reversal, it characterizes the properties of a paramagnetic structure (denoted by G1' in Shubnikov's notation, where G is any space group). However, this classification is not directly relevant for duality because $\mathrm{D}$ can be present in the group of one domain of a phase and absent in another domain (where it is replaced by another possible duality operator $\mathrm{gDg}^{-1}$ ). Thus, it turns out to be more convenient to distinguish between "dual-free groups" (containing only dual-free operations) and "dual groups" (containing dual operations). The unprimed phases (R, C, EL) have dual groups while the primed phases (C', R', R', EL') have dual-free groups. In a phase with a dual group it is always possible to find one domain (by setting the two Goldstone phases to zero) in which $\mathrm{D}$ is 
present. Accordingly, we use the Shubnikov's notation applied to this special domain for denoting the extended symmetry groups of the unprimed phase as $\tilde{G}_{p h}=G_{p h} 1^{1}$ whereas in the primed phase $\tilde{G}_{p h}=G_{p h}$.

Unlike time reversal, D does not commute with all the elements of $\mathrm{G}_{\mathrm{L}}$. It commutes only with the operations $\mathrm{C}_{\phi} \mathrm{T}_{\mathrm{t}}$ provided that $\phi-\mathrm{kt}=\mathrm{n} \pi$ (n integer) and with the twofold axes $\mathrm{U}_{2 \mathrm{x}}$ and $\mathrm{U}_{2 \mathrm{y}}$ (or with a different pair of perpendicular twofold axes if one chooses another definition for $\mathrm{D}$ ). We denote such operations as "self-dual" (they form a group coinciding with that of a cholesteric). For the non selfdual operations $g$ one can thus define their dual conjugate $\mathrm{DgD}(\mathrm{DgD}=\mathrm{g}$ when $\mathrm{g}$ is self-dual, because $\mathrm{D}^{2}$ is the identity operation). This permits one to make a more subtle classification of the lowsymmetry groups that splits them into "self-dual groups" (which contain only self-dual operations), "globally self-dual groups" (such that $\mathrm{D} \tilde{G}_{p h} \mathrm{D}=\tilde{G}_{p h}$ ) and non-self-dual groups. In the latter case $\tilde{G}_{p h}$ is associated with its "dual-conjugate" $\mathrm{D} \tilde{G}_{p h} \mathrm{D}$. Any dual group is automatically globally self-dual. C, C', EL and EL' are self-dual, R is globally self-dual, and R' and R" are non-self-dual phases which are mutually dual conjugated. The analogy between duality and time reversal is correct only for the selfdual groups, since in this case D is present in all the domains of the dual phases. C and EL are then analogous to paramagnetic structures whereas $\mathrm{C}^{\prime}$ and $\mathrm{EL}^{\prime}$ are analogous to ferromagnetics.

In order to discuss the consequences of this classification let us first extend the duality idea to tensor waves. A set of measurable physical quantities transforming as $\Gamma_{a} \otimes \Gamma_{b}\left(\mathrm{a}, \mathrm{b}=_{0}^{+}, 0_{0}^{-}, 1,2,3 \ldots\right)$ is either "self-dual" if $\mathrm{a}=\mathrm{b}$ or associated with its "dual-conjugate" transforming as $\Gamma_{b} \otimes \Gamma_{a}$. For instance, the order-parameter modulus $\left(\rho_{1}^{2}+\rho_{2}^{2}\right.$, which transforms as $\left.\Gamma_{0}^{+} \otimes \Gamma_{0}^{+}\right)$and the chiral index $\chi$ are selfdual scalars, whereas the order parameters $\overrightarrow{\mathrm{P}}(\mathrm{z})$ and $\overrightarrow{\mathrm{A}}(\mathrm{z})$ are self-dual vector waves. On the other hand, the homogeneous polarization $\mathrm{P}_{\mathrm{z}}^{(0)}$ and the homogeneous "tilt vector" $\mathrm{A}_{\mathrm{z}}^{(0)}$ are dual-conjugated scalars. The homogeneous tilt vector in the $\mathrm{x}-\mathrm{y}$ plane $\left(\mathrm{A}_{\mathrm{x}}^{(0)}, \mathrm{A}_{\mathrm{y}}^{(0)}\right)$ is dual-conjugated with the first harmonic (i.e., with wave vector $\vec{k}$ ) of the density wave, whereas the homogeneous polarization $\left(\mathrm{P}_{\mathrm{x}}^{(0)}, \mathrm{P}_{\mathrm{y}}^{(0)}\right)$ is dual-conjugated with the first harmonic of the chirality wave $\chi(\mathrm{z})$. The macroscopic optic tensor $\left(\varepsilon_{\mathrm{xx}}-\varepsilon_{\mathrm{yy}}, \varepsilon_{\mathrm{xy}}\right)$ (second-rank homogeneous tensor) is dual-conjugated with the second harmonic of the density wave.

The main physical consequences of the duality result from the following fact: Whenever a dual-free symmetry $\mathrm{g}$ forbids the existence of a tensor wave (or some of its components) transforming as $\Gamma_{a} \otimes \Gamma_{b}$, its dual-conjugate $\mathrm{DgD}$ forbids (the same components of) $\Gamma_{b} \otimes \Gamma_{a}$. For instance, the inversion I cancels the three components $\mathrm{P}_{\mathrm{z}}^{(0)}$ and $\left(\mathrm{P}_{\mathrm{x}}^{(0)}, \mathrm{P}_{\mathrm{y}}^{(0)}\right)$ of the macroscopic polarization, then the mirror plane $\sigma_{x}=\mathrm{DID}$ cancels the homogeneous tilt $\mathrm{A}_{\mathrm{z}}^{(0)}$ and the first harmonic of the chirality wave $\chi(\mathrm{z})$. Consequently, in a globally self-dual phase the forbidden tensor waves are either self-dual or appear as dual-conjugated pairs. The same property occurs for the allowed tensor waves. Furthermore, allowed dual-conjugated tensor waves have similar thermodynamic properties : They vanish at the same temperature with the same critical exponents. Analogously, in the dual conjugated phases R' and R", whenever a tensor wave is forbidden (or allowed) in one phase, then its dual conjugate is forbidden (or allowed) in the other phase (with the same thermodynamic behavior):

1- In the C-phase the homogeneous polarization $\left(\mathrm{P}_{\mathrm{x}}^{(0)}, \mathrm{P}_{\mathrm{y}}^{(0)}\right)$ and tilt vector $\left(\mathrm{A}_{\mathrm{x}}^{(0)}, \mathrm{A}_{\mathrm{y}}^{(0)}\right)$ are forbidden, then their dual-conjugates, namely the first harmonic of the density wave and of $\chi(\mathrm{z})$, are also forbidden. Moreover, $\mathrm{G}_{\mathrm{C}}$ forbids any homogeneous tensor (except scalars) and, according to the 
previous duality rule, any scalar wave. Thus, $\mathrm{C}$ is macroscopically $2 \mathrm{D}$-isotropic and not smectic : It is optically uniaxial, on the one hand, and it does not give rise to Bragg peaks in normal (non-resonant) $\mathrm{x}$-ray diffraction, on the other hand. Moreover, the order-parameter modulus $\mathrm{n}_{\mathrm{L}}\left(\Gamma_{0}^{+} \otimes \Gamma_{0}^{+}\right)$and the chirality index $\chi\left(\Gamma_{0}^{-} \otimes \Gamma_{0}^{-}\right)$are permitted while the macroscopic longitudinal polarization $\mathrm{P}_{\mathrm{z}}^{(0)}$ $\left(\Gamma_{0}^{+} \otimes \Gamma_{0}^{-}\right)$and tilt vector $\mathrm{A}_{\mathrm{z}}^{(0)}\left(\Gamma_{0}^{-} \otimes \Gamma_{0}^{+}\right)$vanish. Indeed, the helical symmetry of $\mathrm{C}$ makes it "maximally self-dual" because it allows only self-dual tensor waves $\left(\Gamma_{a} \otimes \Gamma_{a}\right)$ and, furthermore, all the self-dual tensor waves. For instance, in the $\mathrm{x}-\mathrm{y}$ plane a single harmonic (with wave vector $\vec{k}$ ) of the polarization $\overrightarrow{\mathrm{P}}(\mathrm{z})$ and tilt $\overrightarrow{\mathrm{A}}(\mathrm{z})$ waves are allowed, hence making the C-phase perfectly helielectric. Similarly, a single harmonic (with wave vector $2 \vec{k})$ of the $2 \mathrm{D}$-optic tensor $\left(\varepsilon_{\mathrm{xx}}(\mathrm{z})-\varepsilon_{\mathrm{yy}}(\mathrm{z}), \varepsilon_{\mathrm{xy}}(\mathrm{z})\right)$ is permitted, yielding the same rotatory-power effect than in a cholesteric phase, and the same optic gap features (within a much shorter wavelength range).

2- C' differs from $\mathrm{C}$ only by the onset of $\mathrm{P}_{\mathrm{z}}^{(0)}$ and $\mathrm{A}_{\mathrm{z}}^{(0)}$ : It is ferroelectric along $\mathrm{Oz}$ because the molecular polarization, which is normal to $\vec{k}$ in $\mathrm{C}$, is uniformly tilted around an axis normal to $\vec{k}$ and to the molecular symmetry axis. On the other hand, several tensor waves with the same transformation properties (as, for instance, $\overrightarrow{\mathrm{A}}(\mathrm{z})$ and $\overrightarrow{\mathrm{P}}(\mathrm{z})$ ), which are locked in parallel directions in $\mathrm{C}$, may have different orientations in C'. For instance, $\overrightarrow{\mathrm{A}}(\mathrm{z})$ and $\overrightarrow{\mathrm{P}}(\mathrm{z})$ are mutually shifted by a constant angle in C'.

3- In the group of the linear R-phase the mirror planes (and inversion) are not self-dual but they appear by pairs of dual-conjugated operations : $\left(\sigma_{z}, \sigma_{y}\right)$ and $\left(I T_{\pi k}, \sigma_{x} T_{\pi k}\right)$. $\mathrm{R}$ is smectic and permits all the even harmonics of the density wave $\left(\Gamma_{0}^{+} \otimes \Gamma_{2 n}\right)$ and all the homogeneous even-rank tensors $\left(\Gamma_{2 n} \otimes \Gamma_{0}^{+}\right)$as for instance the $2 \mathrm{D}$-optic tensor $\left(\varepsilon_{\mathrm{xx}}-\varepsilon_{\mathrm{yy}}, \mathcal{E}_{\mathrm{xy}}\right)$, which makes the phase biaxial. R is also characterized by the onset of all the odd harmonics of $\overrightarrow{\mathrm{P}}\left(\Gamma_{1} \otimes \Gamma_{2 p+1}\right)$, each one giving one resonant Bragg peak at $(2 p+1) \vec{k}$. Simultaneously, the first harmonic of all the odd-rank tensor waves $\left(\Gamma_{2 n+1} \otimes \Gamma_{1}\right)$ are present in $\mathrm{R}$.

The dual features of R' and R" appear clearly as one considers the tensors appearing at the transitions $\mathrm{R} \rightarrow \mathrm{R}$ ' and $\mathrm{R} \rightarrow \mathrm{R}$ '. A homogeneous tilt vector $\mathrm{A}_{\mathrm{z}}^{(0)}$ appears in $\mathrm{R}^{\prime}$ while a homogeneous polarization $\mathrm{P}_{\mathrm{z}}^{(0)}$ appears in $\mathrm{R}^{\prime}$. $\mathrm{A}_{\mathrm{z}}^{(0)}$ indicates that in $\mathrm{R}^{\prime}$ the direction of the transverse polarization (or equivalently of the molecular planes) is not locked, as in $\mathrm{R}$, and can change with temperature. Along the same way, in $\mathrm{R}^{\prime}$ all the even harmonics of the chirality wave $\chi(\mathrm{z})$ appear, whereas their dual conjugates, the homogeneous pseudo tensors of even ranks, appear in R". Moreover, on approaching the $R^{\prime} \rightarrow R$ transition temperature $T^{\prime}$ the amplitude of the $2 n$ 'th harmonic vanishes as $\left(T-T^{\prime}\right)^{n}$, whereas the modulus of the tensor with rank $2 n$ vanishes as $\left(T-T^{\prime \prime}\right)^{n}$ at the $R$ " $\rightarrow R$ transition temperature $T$ ".

3- In EL all the tensor waves permitted in $\mathrm{C}$ and in $\mathrm{R}$ are also permitted. Moreover the odd harmonics of the density wave $\left(\Gamma_{0}^{+} \otimes \Gamma_{2 p+1}\right)$ together with the homogeneous tensors of odd rank $\left(\Gamma_{2 n+1} \otimes \Gamma_{0}^{+}\right)$take non-zero values.

\section{Dual classification of Line Defects}

Line defects are topologically non-trivial fields of the Goldstone angles $\varphi_{T}$ and $\varphi_{R}$ (see Eq.6), on the one hand, and of the direction of $\vec{k}$ on the other hand. We present here below a complete topological classification of the possible defects, using standard homotopy analysis of the image group. It clearly reveals the dual character of the vector-wave theory, for they naturally classify into self-dual 
(dispiration) and pairs of dual conjugated (dislocation/disclination) lines. Nevertheless, one does not expect similar thermodynamic behaviors for dual-conjugated defects since the line core lies in the real space, which does not exhibit the same translation/rotation duality as the order-parameter space. The dual symmetry is a property of the order-parameter space, not of the actual space. It applies to the classification of defects because at the topological level the possible defects are completely determined by the geometry of the Goldstone part of the order-parameter space, which is strongly constrained by the dual symmetry. In particular, we shall see that homotopy group considerations reflect directly its dual structure.

\subsection{Circular phases}

When one considers the condensation of a transverse vector wave in a perfect nematic phase (with $\vec{k}$ parallel to the optic axis) the thermodynamic model presented in sections I and II remains valid, but the direction of $\vec{k}$ is locked even in inhomogeneous ordered states. The same situation occurs when, starting from the isotropic phase, the walls of the sample are prepared in order to lock the direction of $\vec{k}$ (e.g., in homeotropic-like configurations). In these two cases, the inhomogeneous states can result only from the space variation of the angles $\varphi_{T}$ and $\varphi_{R}$. Hence, we shall now distinguish two types of linear defects : Type-1 $(\chi-)$ lines involve variations of $\varphi_{T}$ and $\varphi_{R}$ only. They appear after transitions from either the free isotropic liquid or the homogeneous nematic. Type- $2\left(\lambda_{-}, \lambda_{+}\right)$-lines involve spatial variations of the direction of $\vec{k}$. They cannot be induced by transitions from defect-free nematics or in $\vec{k}$-locked configurations, but only in the isotropic liquid or in nematics with preexisting disclinations.

Figure 3. Line defects in C. The "equal phase" surfaces (analogous to the smectic layers in $\mathrm{R}$ and EL) are represented by continuous lines, and the polarization by arrows when it is parallel to the figure and circles when it is normal to it.. (a) $\chi$-line ( $2 \pi$-disclination) with a core parallel to $\overrightarrow{\mathrm{k}}$. (b) $\chi$-line with a core normal to $\overrightarrow{\mathrm{k}}$ (jelly-roll configuration). A domain wall between two oppositely wound domains is represented by a thick line. (c) $(\lambda-$ ) line $(-\pi$-disclination with core normal to $\overrightarrow{\mathrm{k}})$. (c) $(\lambda+)$ line $(+\pi$-disclination with core normal to $\overrightarrow{\mathrm{k}})$.

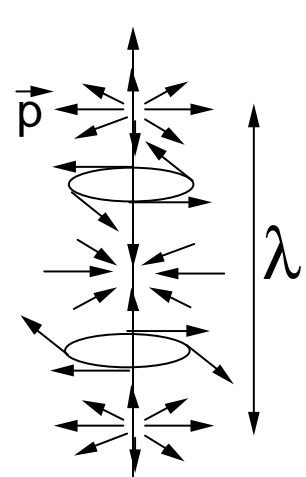

(a)

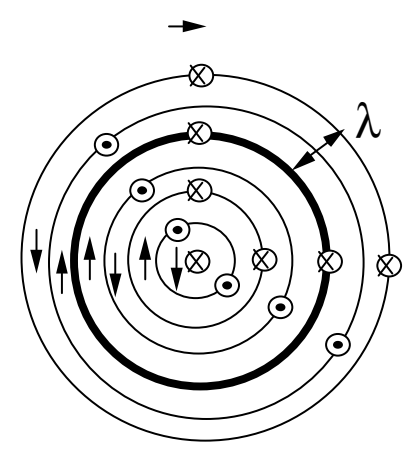

(b)

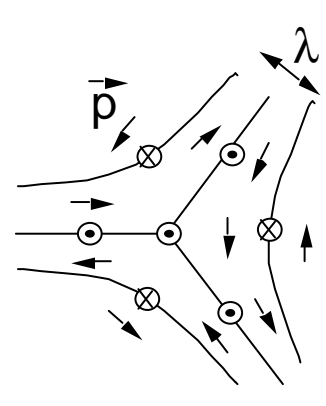

(c)

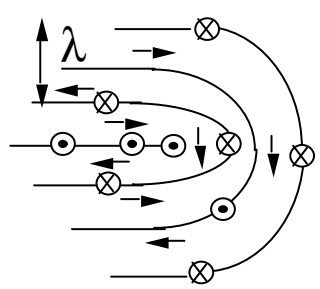

(d)

Type-1: Starting from a homogeneous nematic phase, only $\chi$-lines can take place in C and C'. Their topology is generated by a rotation of the local helix to an angle $2 \pi$ about the line core. The line is parallel to $\vec{k}$ and the polarization turns around it as in Fig. 3a. The order-parameter variations 
around the core can be interpreted within dual point of views: (i) The polarization rotates by an angle $2 \pi$ (disclination line with unit winding number). (ii) The wave is translated by a distance $\lambda$ (dislocation with unit Burgers vector). As long as the direction of $\vec{k}$ is fixed by the underlying homogeneous nematic, an infinity of such defects with winding numbers...,-2,-1,1,2 .. are possible. Contrariwise, when the circular phases arise from the isotropic liquid state the direction of $\vec{k}$ can vary so that disclinations with even winding numbers become topologically trivial, whereas those with odd numbers all become topologically equivalent. Accordingly, there exists in this case a single type of $\chi$ defect.

$\chi$-defects could also appear in the "jelly roll" configuration where $\vec{k}$ is perpendicular to the line (Fig. 3b). In this configuration $\vec{k}$ rotates about the line, transforming the planes of the homogeneous smectic into cylinders wound around the disclination core. It can be associated with cylindrical walls separating domains of opposite handedness. The wave vector $\vec{k}$ remains continuous where it crosses the wall, but inside the cylinder the polarization precesses as a right-handed helix, and as a left-handed one outside. Note that such wall cannot exist in cholesterics since all the domains have the same handedness in chiral systems.

Type-2: To describe the two other defects it is convenient to start from the classification of disclinations in cholesterics. Indeed, a cholesteric phase is analogous to C and C', whose helical groups combine translations with rotations, though the cholesteric local point group is non polar and orthorhombic $\left(\mathrm{D}_{2}\right)$ while it becomes polar and monoclinic $\left(\mathrm{C}_{2}\right)$ in $\mathrm{C}$, and triclinic $\left(\mathrm{C}_{1}\right)$ in $\mathrm{C}^{\prime}$. Thus, although the disclinations are similar in these three phases, four types can be found in cholesterics, while only three types exist in $\mathrm{C}$, and one in $\mathrm{C}^{\prime}$ :

- The $\chi$-line is common to the three phases.

- The remaining cholesteric lines are generated by rotations by angles $\pi$ or $-\pi$ about the three orthogonal axes $\mathrm{x}, \mathrm{y}$ and $\mathrm{z}$. In cholesterics $\pi$ and $-\pi$ rotations are topologically equivalent, yielding three types of defects (x,y and $\mathrm{z}$ ). On the contrary, in $\mathrm{C} \pi$ and $-\pi$ are not equivalent but $\pi$ around $\mathrm{x}$ is equivalent to $\pi$ around y or $\mathrm{z}$, yielding only two distinct defects ( $\pi$ and $-\pi$ ). Their structures, depicted in Figs. 3-c,d, are similar to the cholesteric lines denoted as $\lambda_{+}$and $\lambda_{-}$. Like $\chi$ they can be observed in circular phases appearing in nematics provided that a $\pm \pi$-disclination line preexists. A nematic wedge disclination (angle $2 \pi \mathrm{m}$ ) transforms in $\lambda_{+}$(for $\mathrm{m}=1 / 2$ ) or $\lambda_{-}$(for $\mathrm{m}=-1 / 2$ ) at the Nematic $\rightarrow \mathrm{C}$ transition. $\lambda_{\text {- }}$ and $\lambda_{+}$become topologically unstable in $\mathrm{C}^{\prime}$.

Combining $\chi, \lambda_{\text {- and }} \lambda_{+}$yields the following merging effects: Two lines of the same type annihilate each other. Two lines of different types combine in such a way as to form a single defect of the third type.

\subsection{Linear Phases}

In $\mathrm{R}$ infinitely many distinct type1-defects can be obtained by combining two elementary dispirations: (i) One in which the structure is translated by $\lambda / 2$ along $\mathrm{z}$ and rotated clockwise by an angle $\pi$ when circling around the core, and (ii) its counterclockwise image. Combining (i) with itself yields a pure dislocation with Burgers vector equal to $\lambda$ (two smectic layers). Combining (i) and (ii) (but with opposite translations $+\lambda / 2$ and $-\lambda / 2$ ) yields a pure $2 \pi$-disclination. (i) and (ii) are obviously dual-conjugated. More generally, let us denote the defect (i) by $\{1 / 2,1 / 2\}$ (shorthand for $\left\{\frac{1}{2} \lambda, \frac{1}{2} 2 \pi\right\}$ ) 
and the defect (ii) by $\{1 / 2,-1 / 2\}$. A general type- 1 defect reads then $\{n, p\}$, where $n$ and $p(\geq 0)$ are simultaneously integers or simultaneously half integers. They combine together according to the rule $\{n, p\}\left\{n^{\prime}, p^{\prime}\right\}=\left\{n_{n} n^{\prime}, p^{\prime}+p^{\prime}(\bmod .2)\right\} . \quad\{n, p\} \quad$ is self-dual if $n= \pm p(\bmod .2)$ whereas $\{n, p\}$ and $\{\mathrm{p}, \mathrm{n}(\bmod .2)\}$ are dual conjugated. $\{\mathrm{n}$ (integer), 0$\}$ is a pure dislocation while $\{0,1\}$ is the single pure disclination. Let us finally notice that $\{n, p\}$ and $\{-n, p+1\}$ are topologically equivalent. Four examples of such defects are represented if Figs.4-a,b,c,d.

Four type-2 elementary defects arise when $\vec{k}$ can vary : In (iii/ \pm ), the structure turns by $\pm \pi$ about an axis parallel to the polarization (Figs. 4-f,h). In (iv/ \pm ), the structure turns by $\pm \pi$ about an axis normal to the polarization and to $\vec{k}$ (Figs. $4-\mathrm{e}, \mathrm{g}$ ). They can also be considered as the traces of $\pm \pi$ disclinations in an underlying nematic phase. They combine according to the following rules:

$$
\begin{aligned}
& (\mathrm{iii} /+)(\mathrm{iii} /+)=(\mathrm{iv} /+)(\mathrm{iv} /+)=(\mathrm{iii} /-)(\mathrm{iii} /-)=(\mathrm{iv} /-)(\mathrm{iv} /-)=\{\mathrm{n}, 1\}(\text { or }\{-\mathrm{n}, 0\}) \\
& (\mathrm{iii} /+)(\mathrm{iii} /-)=(\mathrm{iv} /+)(\mathrm{iv} /-)=\{\mathrm{n}, 0\} \quad(\text { or }\{-\mathrm{n}, 1\}) \\
& (\mathrm{iii} /+)(\mathrm{iv} /+)=(\mathrm{iii} /-)(\mathrm{iv} /-)=\{\mathrm{n}+1 / 2,1 / 2\}(\text { or }\{-\mathrm{n}-1 / 2,3 / 2\}) \\
& (\mathrm{iii} /+)(\mathrm{iv} /-)=(\mathrm{iii} /-)(\mathrm{iv} /+)=\{\mathrm{n}+1 / 2,3 / 2\}(\text { or }\{-\mathrm{n}-1 / 2,1 / 2\}) \\
& \{\mathrm{n}, 0\}\{\mathrm{iii} /+\}=\{\mathrm{n}, 1\}\{\mathrm{iii} /-\}=\{\mathrm{n}+1 / 2,1 / 2\}\{\mathrm{iv} /+\}=\{\mathrm{n}+1 / 2,3 / 2\}\{\mathrm{iv} /-\}=\{\mathrm{iii} /+\} \\
& \{\mathrm{n}, 0\}\{\mathrm{iv} /+\}=\{\mathrm{n}, 1\}\{\mathrm{iv} /-\}=\{\mathrm{n}+1 / 2,3 / 2\}\{\mathrm{iii} /+\}=\{\mathrm{n}+1 / 2,1 / 2\}\{\mathrm{iii} /-\}=\{\mathrm{iv} /+\} \\
& \{\mathrm{n}, 0\}\{\text { iii/- }\}=\{\mathrm{n}, 1\}\{\mathrm{iii} /+\}=\{\mathrm{n}+1 / 2,3 / 2\}\{\mathrm{iv} /+\}=\{\mathrm{n}+1 / 2,1 / 2\}\{\mathrm{iv} /-\}=\{\mathrm{iii} /-\} \\
& \{\mathrm{n}, 0\}\{\mathrm{iv} /-\}=\{\mathrm{n}, 1\}\{\mathrm{iv} /+\}=\{\mathrm{n}+1 / 2,1 / 2\}\{\mathrm{iii} /+\}=\{\mathrm{n}+1 / 2,3 / 2\}\{\mathrm{iii} /-\}=\{\mathrm{iv} /-\}
\end{aligned}
$$

where $\mathrm{n}$ may be any integer. The four first association laws leave $\mathrm{n}$ indeterminate in the resulting defect. One can build pure dislocations by associating type- 2 defects with the same nature but different angles [e.g., (iii/+) and (iii/-)], or a pure disclination $\{0,1\}$ with defects having the same nature and angle [e.g., (iii/+) and (iii/+)]. Similarly, the generators (i) and (ii) can arise from combinations involving type-2 defects of opposite nature and the same angle [e.g., (iii/+),(iv/+)] for (i), or opposite angles [e.g., (iii/+),(iv/-)] for (ii). In R' and R" (i), (ii) and their combinations $\{n, p\}$ persist while (iii/ \pm ) and (iv/ \pm ) become unstable by the same process which forbids $\pi$-disclinations in conventional $\mathrm{SmC}$ phases. Moreover $\{\mathrm{n}, \mathrm{p}\}$ and $\{-\mathrm{n}, \mathrm{p}+1\}$ become topologically independent. Hence, the dualconjugated phases $\mathrm{R}$ ' and R" present the same line-defect classes. 
Figure 4. Line defects in R. The straight lines indicate the edge of the smectic layers. (a) Dispiration $\{1 / 2,1 / 2\}$ with core parallel to $\overrightarrow{\mathrm{k}}$. (b) An equivalent defect with core normal to $\overrightarrow{\mathrm{k}}$. (c) Pure $\{0,1\} 2 \pi$-disclination. The arrows and circles indicate the components of the polarization $\overrightarrow{\mathrm{P}}$ at the center of the layers respectively parallel and normal to the figure. (d) Pure $\{1,0\}$ dislocation. (e) $-\pi$-disclination of type (iv,-). (f) $-\pi$ disclination of type (iii,-). (g) $+\pi$-disclination of type (iv,+). (h) $+\pi$-disclination of type (iv/-).

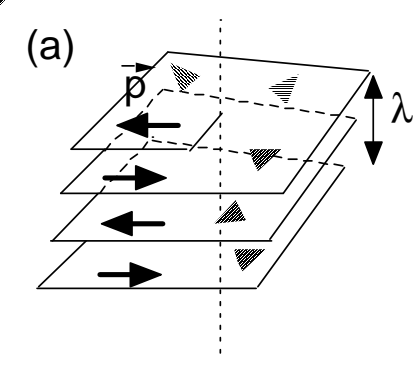

(b)

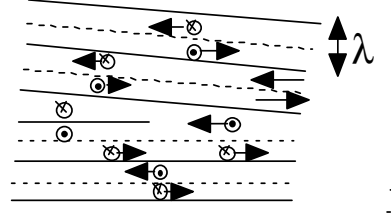

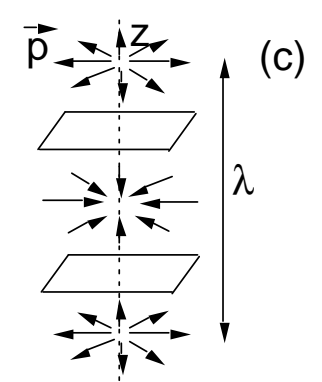

(d)

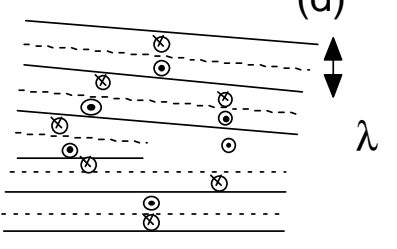

(e)

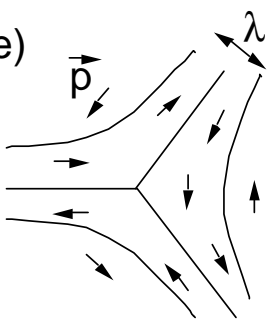

(f)

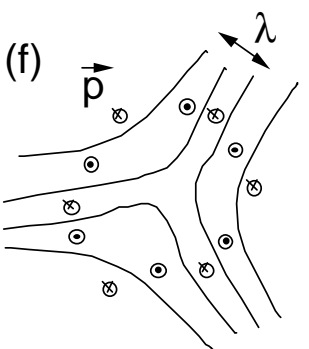

(g)

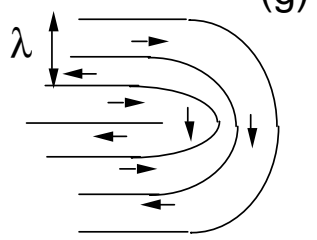

(h)

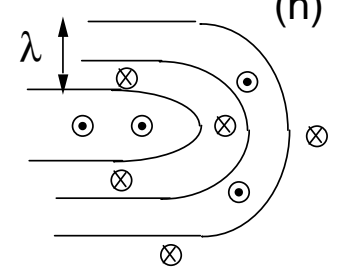

\subsection{Elliptic Phases}

At first sight the classification in EL is the same as that in $\mathrm{R}$, whereas in EL' it is equivalent to that in R' and R'. However, one has yet considered only approximate elliptic structures, in which the two circular polar waves forming the elliptic wave have the same wavelength. In fact, we have shown in references [5] that EL and EL' are actually incommensurate. In EL the polarization wave reads:

$$
\vec{P}(z)=2\left(\rho_{1}+\rho_{2}\right) \cos [K z] \vec{e}_{X}(z)+2\left(\rho_{2}-\rho_{1}\right) \sin [K z] \vec{e}_{Y}(z)
$$

where $\vec{e}_{\mathrm{X}}(\mathrm{z})=\cos (\mathrm{Qz}) \vec{e}_{x}-\sin (\mathrm{Qz}) \vec{e}_{y}$ and $\vec{e}_{\mathrm{Y}}(\mathrm{z})=\sin (\mathrm{Qz}) \vec{e}_{x}+\cos (\mathrm{Qz}) \vec{e}_{y}$ are perpendicular unit vectors turning within the $\mathrm{x}-\mathrm{y}$ plane. The two wavelengths $\lambda_{\text {mod }}=1 / \mathrm{Q}$ and $\lambda=1 / \mathrm{K}<<\lambda_{\text {mod }}$ are incommensurate. The plane containing the polarization of two successive molecules precesses slowly around Oz. This helical structure differs from that in $\mathrm{C}$ and $\mathrm{C}^{\prime}$ in two respects : (i) The length scale of the elliptic helical modulation is much larger than the molecular size (typically within the optic range) while the scale of the pitch $\lambda$ in the circular structures ranges within molecular length. (ii) The helical symmetry in $\mathrm{C}$ and $\mathrm{C}^{\prime}$ is continuous (perfect helix) while it is discrete in EL and EL'. However, the symmetry groups of EL and EL' are not broken by the incommensurate modulations. Indeed, Eq. (10) shows that the modulated space groups are generated by the screw axis $T_{\lambda / 2} C_{\pi-Q \lambda / 2}$ (which becomes $2_{1}$ when $\mathrm{Q}=0$ ) and $\mathrm{U}_{2 \mathrm{x}}$. The groups with $\mathrm{Q}=0$ and $\mathrm{Q} \neq 0$ are isomorphic. Accordingly, the classification of line defects is not modified by the modulation though their spatial structures are changed. 


\section{Analogy With d-Wave Superconductivity}

Attracting fermions in two-dimensional systems condense at low temperature into bound states, the so called Cooper pairs [24]. These states may be classified according to their angular and spin moments [26-28]. For instance, in a conventional superconductor two electrons form a charged Cooper pair with zero orbital and spin moments. These «s-wave» pairs are responsible on the specific properties (zero resistivity, magnetic flux expulsion and Josephson effect) of the superconducting state. At the symmetry point of view superconductors are characterized by the breakdown of the electromagnetic gauge symmetry [29]. Such gauge operations, denoted by $\mathrm{g}_{\alpha}$, transform the Cooper pair wave-function $\psi$ into equivalent wave functions $\exp (\mathrm{i} \alpha) \psi$. In the superconducting state $\psi$ is different from zero and the gauge symmetries are broken. However, since the s-wave function is isotropic it does not break the rotational symmetry. On the contrary, the wave function can break spatial symmetries when its orbital momentum is different from zero. Such a phenomenon occurs in various superfluid systems, such as the superfluid $\mathrm{He}_{3}$ (p-wave pairing) [26,27,28], the heavy fermions (p or d-wave pairing) [30] and the high- $\mathrm{T}_{\mathrm{c}}$ oxide superconductors (d-wave pairing) [31,32]. We shall show now that the theory of d-wave $2 \mathrm{D}$ superconductors is strongly analogous to the theory of bentcore liquid crystals.

Let $S_{1 z}$ and $S_{2 z}$ be the spins of two fermions at positions $\vec{r}_{1}$ and $\vec{r}_{2}$, and $\psi\left(r, \theta, S_{1 z}, S_{2 z}\right)$ their wave function. $\mathrm{r}$ and $\theta$ are the polar coordinates of the relative position $\vec{r}_{1}-\vec{r}_{2}$. In a d-wave the orbital momentum is $\mathrm{L}=2$, and the spin is in the singlet state $\mathrm{S}\left(\mathrm{S}_{1 \mathrm{z}}, \mathrm{S}_{2 \mathrm{z}}\right)$ [15]:

$$
\psi\left(\mathrm{r}, \theta, \mathrm{S}_{1 \mathrm{z}}, \mathrm{S}_{2 \mathrm{z}}\right)=\left\{\mathrm{D}_{+} \exp (2 \mathrm{i} \theta)+\mathrm{D}_{-} \exp (-2 \mathrm{i} \theta)\right\} \mathrm{S}\left(\mathrm{S}_{1 \mathrm{z}}, \mathrm{S}_{2 \mathrm{z}}\right)
$$

$\mathrm{D}_{+}=\left|\mathrm{D}_{+}\right| \exp \left(\mathrm{i} \phi_{+}\right), \mathrm{D}_{-}=\left|\mathrm{D}_{-}\right| \exp \left(\mathrm{i} \phi_{-}\right), \mathrm{D}_{+} *$ and $\mathrm{D}_{-} *$ are the four complex components of the order parameter. They transform according to an irreducible representation of the normal phase symmetry group, which contains the gauge transformations $\mathrm{g}_{\alpha}$, the continuous $2 \mathrm{D}$ rotations $\mathrm{C}_{\varphi}$, as well as the discrete time reversal $\mathrm{T}$ and mirror plane $\sigma_{\mathrm{x}}$ operations. The corresponding matrices are given by:

$$
\begin{aligned}
& g_{\alpha}=\left(\begin{array}{llll}
e^{\mathrm{i} \alpha} & & & \\
& e^{-\mathrm{i} \alpha} & & \\
& & e^{\mathrm{i} \alpha} & \\
& & & e^{-\mathrm{i} \alpha}
\end{array}\right), C_{\varphi}=\left(\begin{array}{llll}
e^{2 \mathrm{i} \varphi} & & & \\
& e^{-2 \mathrm{i} \varphi} & & \\
& & e^{-2 \mathrm{i} \varphi} & \\
& & & e^{2 \mathrm{i} \varphi}
\end{array}\right)
\end{aligned}
$$

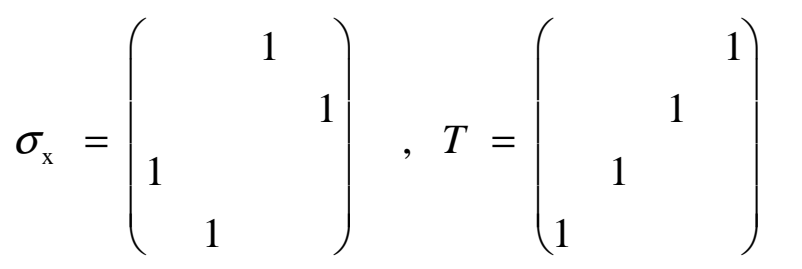

in the basis $\left\{\mathrm{D}_{+}, \mathrm{D}_{+} *, \mathrm{D}_{-}, \mathrm{D}_{-} *\right\}$. These matrices generate the "image group":

$$
\mathrm{O}_{\mathrm{G}}(2) \otimes \mathrm{O}_{\mathrm{R}}(2)
$$


where $\mathrm{O}_{\mathrm{G}}(2)$ contains gauge and time reversal transformations whereas $\mathrm{O}_{R}(2)$ is the 2D rotation group $\mathrm{C}_{\infty \mathrm{V}}$. As in the vector wave model $\mathrm{O}_{\mathrm{G}}(2)$ and $\mathrm{O}_{\mathrm{R}}(2)$ are isomorphic copies of the $2 \mathrm{D}$ orthogonal group $\mathrm{O}(2)$ (see Eq. (7)). One sees immediately that the order parameter (12) transforms as the irreducible representation $\Gamma_{1}{ }^{(\mathrm{G})} \otimes \Gamma_{1}{ }^{(\mathrm{R})}$ of $\mathrm{O}_{\mathrm{G}}(2) \otimes \mathrm{O}_{\mathrm{R}}(2)$. The analogy between the bent-core and d-wave models results from, (i) the group isomorphism of their high-symmetry phases (see Eqs. 7 and 13), (ii) the fact that in both cases the order parameter spans the irreducible representation $\Gamma_{1} \otimes \Gamma_{1}$ of these groups. Consequently, the d-wave model [15] exhibits a rotation/gauge duality completely analog to the translation/rotation duality of bent-core mesophases.

In order to realize concretely the analogy we may identify the image groups of the two systems along two ways. In the first way the rotation group of the liquid crystal is identified with the rotation group of the superfluid whereas the translation group is identified with the gauge group. In the second way the identifications are reversed. These cases are obviously dual-conjugated. More precisely :

First analogy : A rotation of the liquid crystal by an angle $\varphi$ is equivalent to a rotation of the superfluid by an angle $\varphi / 2$. A translation of $\Delta \mathrm{z}$ along the helical axis is equivalent to a gauge transformation by an angle $\alpha=\mathrm{k} \Delta \mathrm{z}$ (as in the deGennes' analogy for Sm-A [19,21]). Space inversion I is equivalent to time reversal $\mathrm{T}$, and the liquid crystal mirror plane $\sigma_{\mathrm{x}}$ is equivalent to the superfluid mirror $\sigma_{\mathrm{x}}$. This yields the following identification between the order-parameters:

$$
\mathrm{D}_{-} \leftrightarrow \eta_{2}, \mathrm{D}_{+} \leftrightarrow-\eta_{1} *
$$

Second analogy : In the second identification the rotational properties of the superconductor are equivalent to the translational properties of the liquid crystal (as in the deGennes' analogy for Sm-C $[20,21])$ :

$$
\begin{aligned}
& \mathrm{C}_{\varphi} \leftrightarrow \mathrm{g}_{\alpha}(\alpha=\varphi), \sigma_{\mathrm{x}} \leftrightarrow \mathrm{T} \\
& T_{t} \leftrightarrow \mathrm{C}_{\varphi}(\varphi=\mathrm{kt} / 2), I \leftrightarrow \sigma_{\mathrm{x}} \\
& \mathrm{D}_{-} \leftrightarrow-\eta_{2} *, \mathrm{D}_{+} \leftrightarrow \eta_{1}^{*}
\end{aligned}
$$

Accordingly, the physical (i.e., gauge-invariant) properties of d-wave Cooper pairs identify with either liquid crystal translational properties or with their dual rotational analogs.

This comparison allows us to expose the properties of superconducting phases by simply translating those studied in bent-core phases. However, the analogy is actually complete when one defines a secondary wave function, analog to $\overrightarrow{\mathrm{A}}(\mathrm{z})$, with the same symmetry properties as $\psi\left(\mathrm{r}, \theta, \mathrm{S}_{1 \mathrm{z}}, \mathrm{S}_{2 \mathrm{z}}\right)$. This can be achieved in many different ways, one of them being described in Ref. [14] within the context of s-wave superconductivity. Thus, the four unprimed phases, which have already been calculated with a single wave function in Ref. [15], can then be complemented by four additional primed phases. Their properties are summarized in Tables $2-a, b$, and compared with their bent-core analogs. The gauge part of the symmetry groups are not indicated in these tables. Moreover, in systems containing a mirror plane parallel to $\mathrm{x}, \mathrm{y}$ (for instance in high- $\mathrm{T}_{\mathrm{c}}$ superconductors), this plane is never broken by the order parameter, and it must be included in the ordered symmetry groups. For instance, the magnetic symmetry of phase II becomes $4 \mathrm{mmm1}$ '. 
Table 2a. Symmetry and properties of the superconducting unprimed phases. Their analog mesophases are indicated in rows 4 (first analogy) and 5 (second analogy).

\begin{tabular}{|l|l|l|l|l|}
\hline Unprimed phases & Normal & I & II & III \\
\hline Magnetic groups & $\infty \mathrm{m} 1^{\prime}$ & $\infty \mathrm{m}^{\prime}$. & $4 \mathrm{~mm} 1^{\prime}$ & $4 \mathrm{~m}^{\prime} \mathrm{m}^{\prime}$ \\
\hline Properties & $\begin{array}{l}\text { non magnetic } \\
\text { non chiral }\end{array}$ & $\begin{array}{l}\text { magnetic } \\
\text { non chiral }\end{array}$ & $\begin{array}{l}\text { non magnetic } \\
\text { non chiral }\end{array}$ & $\begin{array}{l}\text { magnetic } \\
\text { non chiral }\end{array}$ \\
\hline First analogy & Isotropic & C & R & EL \\
\hline Second analogy & Isotropic & C & R & EL \\
\hline
\end{tabular}

Table 2b. Symmetry and properties of the superconducting primed phases. Their analog mesophases are indicated in rows 4 (first analogy) and 5 (second analogy).

\begin{tabular}{|l|l|l|l|l|}
\hline Primed phases & I' $^{\prime}$ & II' & II" & III' \\
\hline Magnetic groups & $\infty$ & $4 \mathrm{~mm}$ & $41^{\prime}$ & 4 \\
\hline Properties & $\begin{array}{l}\text { magnetic } \\
\text { chiral }\end{array}$ & $\begin{array}{l}\text { magnetic } \\
\text { non chiral }\end{array}$ & $\begin{array}{l}\text { non magnetic } \\
\text { chiral }\end{array}$ & $\begin{array}{l}\text { magnetic } \\
\text { chiral }\end{array}$ \\
\hline First analogy & $\mathrm{C}^{\prime}$ & $\mathrm{R}^{\prime}$ & $\mathrm{R}^{\prime \prime}$ & EL' \\
\hline Second analogy & $\mathrm{C}^{\prime}$ & $\mathrm{R}^{\prime \prime}$ & $\mathrm{R}^{\prime}$ & EL' \\
\hline
\end{tabular}

Phase III and EL help us to illustrate some aspects of the analogy physical meaning. Indeed, the phase-III wave function can be understood as a quantum superposition of Cooper pairs with orbital moments oriented toward $+\mathrm{z}$ and pairs oriented toward $-\mathrm{z}$. Likewise, the elliptic phase results from the superimposition of two circularly polarized waves of opposite handedness. In II the up and down pairs arise with the same amplitude so that the total magnetic moment vanishes. In the analog linear $\mathrm{R}$ phase the right and left helices have the same amplitude, so that there is no winding of the polarization. On the other hand, in I all the pairs are up and the magnetization is maximum, whereas in its analog $\mathrm{C}$ an helix with a single handedness is present so that the chirality is maximum.

The dual classification of physical quantities has a different interpretation with the superconductors. Indeed, the representation $\Gamma_{\mathrm{n}} \otimes \Gamma_{\mathrm{p}}$ can be associated with an observable only when $\mathrm{n}=0$ since only gauge-invariant quantities are measurable. Thus, we have only to consider the quantities $\Gamma_{0}^{+} \otimes \Gamma_{0}^{+}, \Gamma_{0}^{+} \otimes \Gamma_{0}^{-}, \Gamma_{0}^{+} \otimes \Gamma_{\mathrm{p}}$, which represent scalars, pseudo scalars and p-rank tensors invariant under time reversal, on the one hand, and their antisymmetric time-reversal counterparts $\Gamma_{0}^{-} \otimes \Gamma_{0}^{+}, \Gamma_{0}^{-} \otimes \Gamma_{0}^{-}, \Gamma_{0}^{-} \otimes \Gamma_{\mathrm{p}}$, on the other hand. Their values in the ordered phases can be deduced from Table 1 on using any one of the previous analogies (being careful that a tensor of rank $\mathrm{p}$ in liquid crystal is associated with a tensor of rank $2 p$ in superconductors!).

Let us now consider the lowest-degree tensors:

a) Scalars : Pair density $\mathrm{n}_{\mathrm{S}}$, $\mathrm{z}$ projection of the orbital momentum $\mathrm{L}_{\mathrm{Z}}$ and $\mathrm{z}$ projection of an axial vector $\mathrm{A}_{\mathrm{z}}$. The scalar $\sigma$ antisymmetric under time-reversal is the response coefficient associated with a second-order magneto-electric effect : $\overrightarrow{\mathrm{P}}=\sigma \overrightarrow{\mathrm{E}} \times \overrightarrow{\mathrm{B}}$. In structures where $\sigma$ is finite a polarization $\overrightarrow{\mathrm{P}}$ 
perpendicular to the electric field $\overrightarrow{\mathrm{E}}$ appears when a magnetic field $\overrightarrow{\mathrm{B}}$ is applied. This effect happens for instance in unconventional s+s' superconductors [21].

b) Second-rank tensors : Dielectric tensor: [ $2 \varepsilon_{\mathrm{xy}}, \varepsilon_{\mathrm{xx}}-\varepsilon_{\mathrm{yy}}$ ], and a second-rank tensor antisymmetric under time-reversal [ $2 \tau_{\mathrm{xy}}, \tau_{\mathrm{xx}}-\tau_{\mathrm{yy}}$ ]. The $3 \mathrm{D}$ tensor $\tau$ is the response function of the linear magnetoelectric effect $\overrightarrow{\mathrm{P}}=\tau \overrightarrow{\mathrm{B}}$ where $\overrightarrow{\mathrm{P}}$ is the polarization and $\overrightarrow{\mathrm{B}}$ the magnetic field.

c) Forth-rank tensors : $[\mathrm{A}, \mathrm{B}]$ on the one hand, and a fourth-rank tensor antisymmetric under time reversal [A',B']. The latter tensor may represent the elastic $\left(\mathrm{u}_{\mathrm{ij}}\right)$ response $\left(\mathrm{M}_{\mathrm{ijkl}}\right)$ to a bilinear magnetoelectric excitation: $\mathrm{u}_{\mathrm{ij}}=\mathrm{M}_{\mathrm{ijkl}} \mathrm{B}_{\mathrm{k}} \mathrm{E}_{\mathrm{l}}$. Where $\mathrm{A}^{\prime}=\mathrm{M}_{1111}-\mathrm{M}_{2211}-\mathrm{M}_{1122}+\mathrm{M}_{2222}$, and $\mathrm{B}^{\prime}=\mathrm{M}_{1112}-\mathrm{M}_{2212}-$ $\mathrm{M}_{1121}+\mathrm{M}_{2221}$.

The superconducting tensors and their liquid-crystal analogs are indicated in Table 3 . As an example, the bilinear magneto-electric coefficient $\sigma$ in the superconducting phase has two analogs, a homogeneous longitudinal polar vector $\mathrm{P}_{\mathrm{Z}}$ and a homogeneous longitudinal axial vector $\mathrm{A}_{\mathrm{Z}}$. Note again that according to the first analogy liquid-crystal tensors of rank $\mathrm{r}$ are associated with superconducting tensors of rank 2r. For instance, the fourth-rank elastic coefficients in the superconducting system are associated with the second-rank optical tensor in the liquid crystal.

Table 3. Superconducting tensors and their liquid crystal analogs. The type of tensor wave is indicated in the first column. The first number indicates the wave vector and the second number indicates the tensor rank of the waves.

\begin{tabular}{|l|l|l|l|}
\hline Tensors & Superconductor & First analogy & Second analogy \\
\hline$\left[0^{+}, 0^{+}\right]$ & Pair density, $\mathrm{n}_{\mathrm{S}}$ & $\mathrm{n}_{\mathrm{L}}$ & $\mathrm{n}_{\mathrm{L}}$ \\
\hline$\left[0^{+}, 0^{-}\right]$ & Bilinear Mag-elec. $\varepsilon_{1}$ & Polarization $\mathrm{P}_{\mathrm{z}}$ & Axial-vector, $\mathrm{A}_{\mathrm{z}}$ \\
\hline$\left[0^{-}, 0^{+}\right]$ & Linear Mag-elec. $\tau_{1}$ & Tilt-vector $\mathrm{A}_{\mathrm{z}}$ & Polarization $\mathrm{P}_{\mathrm{z}}$ \\
\hline$\left[0^{-}, 0^{-}\right]$ & Magnetization $\mathrm{L}_{\mathrm{z}}$ & Chirality, $\chi$ & Chirality $\chi$ \\
\hline$\left[0^{+}, 1\right]$ & $\begin{array}{l}\text { Biaxiality } \\
{\left[\varepsilon_{\mathrm{xy}}, \varepsilon_{\mathrm{xx}}-\varepsilon_{\mathrm{yy}}\right]}\end{array}$ & $\begin{array}{l}\text { Tilt } \\
\left(\mathrm{T}_{\mathrm{x}}, \mathrm{T}_{\mathrm{y}}\right)\end{array}$ & $\begin{array}{l}\text { Smecticity } \\
\text { Density wave }(\mathbf{k})\end{array}$ \\
\hline$\left[0^{-}, 1\right]$ & $\begin{array}{l}\text { Linear Mag.electric } \\
\left.\tau_{\mathrm{xy}}, \tau_{\mathrm{xx}}-\tau_{\mathrm{yy}}\right]\end{array}$ & $\begin{array}{l}\text { Polarization } \\
\left(\mathrm{P}_{\mathrm{x}}, \mathrm{P}_{\mathrm{y}}\right)\end{array}$ & $\mathrm{A}_{\mathrm{z}} \mathrm{e}^{ \pm \text {ikz }}$ \\
\hline$\left[0^{+}, 2\right]$ & $\begin{array}{l}\text { Tetragonality } \\
\text { Elastic }[\text { A,B] }\end{array}$ & $\begin{array}{l}\text { Biaxiality } \\
\text { Optic } \varepsilon_{\mathrm{xy}}, \varepsilon_{\mathrm{xx}}-\varepsilon_{\mathrm{yy}}\end{array}$ & $\begin{array}{l}\text { Smecticity } \\
\text { Density wave }(2 \mathbf{k})\end{array}$ \\
\hline$\left[0^{-}, 2\right]$ & $\begin{array}{l}3^{\mathrm{d}} \text { order Mag.elect. } \\
{\left[\mathrm{A}^{\prime} \mathrm{B}^{\prime}\right]}\end{array}$ & $\begin{array}{l}\text { Electro-clinic. } \\
{\left[\varepsilon_{x x}^{C}-\varepsilon_{y y}^{C}, \varepsilon_{x y}^{C}\right]}\end{array}$ & $\mathrm{A}_{\mathrm{z}} \mathrm{e}^{ \pm 2 \text { ikz }}$ \\
\hline
\end{tabular}

As with bent-core mesophases, four physical quantities quadratic in the order-parameter components are sufficient to characterize most of the physical properties in the unprimed phases : $\mathrm{n}_{\mathrm{S}}=\left|\mathrm{D}_{+}\right|^{2}+\left|\mathrm{D}_{-}\right|^{2}$ represents the Cooper pair density $\left(\Gamma_{0}^{+} \otimes \Gamma_{0}^{+}\right)$. 
$\mathrm{L}_{\mathrm{z}}=\left|\mathrm{D}_{+}\right|^{2}-\left|\mathrm{D}_{-}\right|^{2}$ represents the $2 \mathrm{D}$ orbital momentum $\left(\Gamma_{0}^{-} \otimes \Gamma_{0}^{+}\right)$.

$\mathrm{A}=\mathrm{D}_{+}{ }^{*} \mathrm{D}_{-}+\mathrm{D}_{-}{ }^{*} \mathrm{D}_{+}, \mathrm{B}=\mathrm{i}\left(\mathrm{D}_{+}{ }^{*} \mathrm{D}_{-}+\mathrm{D}_{-}{ }^{*} \mathrm{D}_{+}\right)$form a quadrivalent tensor $\left(\Gamma_{0}^{+} \otimes \Gamma_{2}\right)$, which can be used to characterize the angular variation of the pair wave function. Indeed, let us write the gaugeinvariant norm of $\psi$ as:

$$
\psi \psi^{*}=n_{S}+A \cos 4 \theta-B \sin 4 \theta
$$

This expression permits one to draw the wave function in each unprimed phase (Fig. 2b). It is circular in the isotropic phase I whereas it presents four branches in the other phases. In phase II the wave function vanishes along four directions. Along the corresponding directions in the reciprocal space nodes appear in the quasiparticle excitation spectrum. This means that when the wave vector $\overrightarrow{\mathrm{k}}$ of a quasiparticle lies parallel to this direction, its energy vanishes continuously as $\overrightarrow{\mathrm{k}}$ get closer to the Fermi surface, while a gap is present in general directions. The occurrence of such nodes results from specific symmetry operations in the phase-II group, and yields most of the unconventional dynamic and thermodynamic properties of this phase [26-28].

A superconductor with four nodes in the excitation spectrum gap (phase II) is analog to a linearlypolarized vector wave (R-phase). The relation between nodes in the superconducting gap and zeroes of the polar wave is reinforced by comparing the four-leave curves (Eq.15) representing $\psi \psi^{*}$ in phase II (Fig.2b) and the bean-shaped curve $\mathrm{M}(\mathrm{z})$ (Eq.8) summarizing the translation properties of the polarization wave in the R-phase (Fig.2a). In the latter, only two leaves are present instead of four in its superconducting analog. This inessential difference results from the second-rank tensor angular character of a d-wave Cooper pair $v s$. first-rank of the polarization wave.

The analogy between nodes in the excitation spectrum and zeroes of the polar wave in phases II and $\mathrm{R}$ is a consequence of their common symmetry features. For instance, from analogy 2 the linear character of the vector-wave (due to the mirror plane $\sigma_{\mathrm{x}}$ ) is related to the paramagnetic feature of phase II (due to the time reversal T). Let us consider one domain of phase II defined by $\varphi_{+}=\varphi_{-}=0$. Its symmetry group is generated by $\mathrm{g}_{\pi} \mathrm{C}_{\pi / 2}, \mathrm{~T}$ and $\sigma_{\mathrm{x}} \cdot \mathrm{g}_{\pi} \mathrm{C}_{\pi / 2}$ changes the sign of the wave function after a $90^{\circ}$ rotation, therefore $\psi(\theta)$ must vanish along four directions. This is the symmetry origin of the four nodes occurring in the gap. In order to determine the vanishing directions let us consider the symmetry $\mathrm{g}_{\pi} \mathrm{C}_{\pi / 2} \sigma_{\mathrm{x}}=\mathrm{g}_{\pi} \sigma_{\mathrm{xy}}$, which changes the sign of the wave function together with a mirror plane directed along $\mathrm{x}+\mathrm{y}$, which transforms $\theta$ into $\pi / 2-\theta: \mathrm{g}_{\pi} \sigma_{\mathrm{xy}} \psi(\theta)=-\psi(\pi / 2-\theta)$. The invariance of $\psi$ under $\mathrm{g}{ }_{\pi} \sigma$ xy provides immediately the four nodes directions: $\psi(\pi / 4)=\psi(3 \pi / 4)=\psi(5 \pi / 4)=\psi(7 \pi / 4)=0$. The zeroes of the vector-waves result from the same arguments. According to the second analogy the generators of the R-phase are $\mathrm{C}_{\pi} \mathrm{T}_{\lambda / 2}, \sigma_{\mathrm{x}}$ and $\mathrm{I}$. $\mathrm{C}_{\pi} \mathrm{T}_{\lambda / 2}$ means that the transverse vector-waves reverse their directions after a translation of $\lambda / 2 . \sigma_{\mathrm{x}}$ indicates that $\overrightarrow{\mathrm{P}}(\mathrm{z})$ is polarized in the (y-z)-plane and $\overrightarrow{\mathrm{A}}(\mathrm{z})$ in the (x,z)-plane. Since the transverse axial and polar vector-waves are linearly polarized and reverse their directions after $\lambda / 2$, they must vanish on a lattice of points separated by $\lambda / 2$. To determine the positions of these zeroes, let us consider the mirror plane $\mathrm{C}_{\pi} \mathrm{T}_{\lambda / 2} \mathrm{I}$ normal to $\mathrm{Oz}$ and located at $\mathrm{z}=\lambda / 4$. This plane forces $\overrightarrow{\mathrm{A}}$ to vanish at $\mathrm{z}=\lambda / 4$ and $3 \lambda / 4$. On the contrary, the zeroes of $\overrightarrow{\mathrm{P}}$ are determined by the location of the inversion centers I located at $\mathrm{z}=0$ and $\lambda$ (analogy 2 makes apparently $\psi$ and $\overrightarrow{\mathrm{A}}$ closer than $\psi$ and $\overrightarrow{\mathrm{P}}$, because of 
the sign change $D_{-} \leftrightarrow-\eta_{2} *$ in the correspondences (13), a $\pi / 2$ phase shift appears between $\overrightarrow{\mathrm{P}}(\mathrm{z})$ and $\psi(\theta)$ as we introduce the additional correspondence $\mathrm{kz} \rightarrow \theta)$.

In conclusion, let us note that in high-Tc superconductors (other superconductors or superfluid are either 3D or do not exhibit d-wave pairing) the exact symmetry is difficult to obtain experimentally (because it involves gauge transformations) and the phase can be identified by indirect gap measurements. In the experimental literature, only the most symmetric phases (I and II) are discussed. As usually in the theory of phase transitions, the least symmetric phases are much less likely, so that we do not really expect that all the predicted phases be actually stabilized."

\section{Conclusion}

To summarize, we have shown that the vector-wave order parameter of bent-core molecules can stabilize eight phases, according to the polarization states and relative orientations of the waves $\vec{A}(\mathrm{z})$ and $\overrightarrow{\mathrm{P}}(\mathrm{z})$. The symmetry groups of these phases contain rotations and translations, which can be interchanged by a dual operation D. D permits one to classify the stable phases, their symmetry groups, and the various tensor waves condensing at the corresponding phase transitions. For instance, the circular $\mathrm{C}$ phase is self dual, i.e., it contains only mixed symmetry operations combining a translation with a rotation, and it allows only the appearance of self-dual tensor waves. The theory of d-wave lamellar high-Tc superconductors presents the same dual character between gauge symmetries and rotations. Moreover, the order parameters and symmetries of the two theories are isomorphic, so that they present strong physical and mathematical analogies. Thus, all the tensor waves of the vector-wave theory have two dual analog tensor quantities in the superconducting system. Each couple of analogs exhibits the same thermodynamic behaviour. We have used analogy and duality to extend the superconductors theory, to classify the possible line defects in bent-core phases, and to discuss the gap in the excitation spectrum of $2 \mathrm{D} d$-wave superconductors.

\section{References}

1. Niori, T.; Sekine, T.; Watanabe, J.; Furukawa; T.; Takezoe, H. Distinct Ferroelectric Smectic Liquid Crystals Consisting of Banana Shaped Achiral Molecules. J. Mat. Chem. 1996, 6, 12311233.

2. Pelzl, G.; Diele, S.; Weissflog, W. Banana Shaped Compounds, a New field of Liquid Crystrals. Adv. Mater. 1999, 11, 707-724.

3. Link, D. R.; Natale, G.; Shao, R.; Maclennan, J. E.; Clark, N. A.; Korblova, E.; Walba, D. M. Spontaneous Formation of Macroscopic Chiral Domains in a Fluid Smectic Phase of Achiral Molecules. Sciences 1997, 278, 1924-1927.

4. Brand, H. R.; Cladis, P. E.; Pleiner, H. Macroscopic Properties of Smectic Liquid Crystals. Eur. Phys. J. B. 1998, 3, 347-353.

5. Kats, E.I.; Lajzerowicz, J. Coupling Between Polarization, Orientation, and Chirality in Smectic Films Formed by Bent-Shaped Molecules. Zh. Eksp. Teor. Fiz. 2000, 117, 951-959 [JETP 2000, 90, 826] (e-print arXiv.cond-mat/9912486). 
6. Roy, A.; Madhusudana, N. V.; Tolédano, P.; Figueiredo Neto, A. M. Longitudinal Spontaneous Polarization and Longitudinal Electroclinic Effect in Achiral Smectic Phases with Bent-Shaped Molecules. Phys. Rev Lett. 1999, 82, 1466-1469.

7. Lorman, V.L.; Mettout, B. Unconventional Mesophases Formed by Condensed Vector Waves in a Medium of Achiral Molecules. Phys. Rev. Lett. 1999, 82, 940-943;

8. Lorman, V.L.; Mettout, B. Theory of Chiral Periodic Mesophases Formed from an Achiral Liquid of Bent-Core Molecules. Phys. Rev. E 2004, 69, 061710.

9. Mettout, B. Theory of Two- and Three-Dimensional Bent-Core Mesophases. Phys. Rev. E 2007, 75, 011706.

10. Brand, H.R.; Cladis, P.; Pleiner, H. Symmetry and Defects in the $\mathrm{C}_{\mathrm{M}}$ Phase of Polymeric Liquid Crystals. Macromolecules 1992, 25, 7223-7226.

11. Eremin, A.; Diele, S.; Pelzl, G.; Nadasi, H.; Weissflog, W.; Salfetnikova, J.; Kresse, H. Experimental Evidence For an Achiral Orthogonal Biaxial Smectic Phase Without In-Plane Order Exhibiting Antiferroelectric Switching Behavior. Phys. Rev. E 2001, 64, 051707.

12. Weissflog, W.; Lischka, C.; Benné, I.; Scharf, T.; Pelzl, G.; Diele, S.; Kruth, H.; Wirth, I. Structure and Properties of Liquid Crystalline Phases Formed by Achiral Banana-Shaped Molecules. Ferroelectrics 1998, 212, 169-177.

13. Macdonald, R.; Kentischer, F.; Warnick, P.; Heppke, G. Antiferroelectricity and Chiral Order in New Liquid Crystals of Nonchiral Molecules Studied by Optical Second Harmonic Generation. Phys. Rev. Lett. 1998, 81, 4408-4411.

14. Mettout, B.; Tolédano, P.; Lorman, V.L. Unconventional $s$-Pairing Wave Superconductivity. Phys. Rev. Lett. 1996, 77, 2284-2287.

15. M. Gufan, Yu.; Vereshkov, G.M. ; Tolédano, P.; Mettout, B.; Bouzerar, R.; Lorman, V.L. Orderparameter Symmetries, Phase Diagrams, and Physical Properties of Two-Dimensional Unconventional Superconductors. I. $d$-Wave-Pairing Superconductivity. Phys. Rev. B 1995, 51, 9219-9227.

16. Tolédano, J. C.; Tolédano, P. The Landau Theory of Phase Transitions; World Scientific: Singapore, HK, 1987.

17. Landau, L. D. Collected Papers; Pergamon Press: Oxford, UK, 1965.

18. Gufan, Yu. M. Structural Phase Transitions; Nauka: Moscow, Russia, 1982.

19. deGennes, P. G. An Analogy Between Superconductors and Smectics-A. P. G. Solid State Commun. 1972, 10, 753-756.

20. deGennes, P. G. Sur la Transition Smectic A-Smectic C. C.R. Acad. Sci. (Paris) B 1972, 274, 758.

21. deGennes, P. G.; Prost, J. The Physics of Liquid Crystals; Clarendon: Oxford, UK, 1993.

22. Renn, S. R.; Lubensky, T. C. Abrikosov Dislocation Lattice in a Model of the Cholesteric-toSmectic-A Transition. Phys. Rev. A 1988, 38, 2132-2147.

23. Renn, S. R.; Lubensky, T. C. Twist-Grain-Boundary Phases Near the Nematic-Smectic- $A-$ Smectic-C Point in Liquid Crystals. Phys. Rev. A 1990, 41, 4392-4401.

24. Bardeen, J.; Cooper, L. N.; Schrieffer, J.R. Theory of Superconductivity. Phys. Rev. 1957, 108, 1175-1204.

25. deGennes, P. G. Superconductivity of Metal and Alloys; Addison-Wesley: New York, NY, USA 1989. 
26. Lifshitz, E. M.; Pitaevskii, L.P. Landau and Lifshitz Course on Theoretical Physics, Vol. 9, §54; Pergamon Press: Oxford, UK, 1980.

27. Anderson, P. W.; Morel, P. Generalized Bardeen-Cooper-Schrieffer States and the Proposed LowTemperature Phase of Liquid He3. Phys. Rev. 1961, 123, 1911-1934.

28. Balian, R.; Werthamer, N.R. Superconductivity with Pairs in a Relative $p$ Wave. Phys. Rev. 1963, 131, 1553-1564.

29. Ginzburg, V. L.; Landau, L. D. On the Theory of Superconductivity. Zh. Eksperim. i Teor. Fiz., 1950, 20, 1064-1082 (Russian); English translation in D.Per Haar and L.D.Landau. Men of Physics, Vo1.1; Pergamon Press, Oxford, 1965, pp.138-167.

30. MacLaughlin, D.E.; Tien, C.; Clark, W. G.; Lan, M. D.; Fisk, Z.; Smith, J. L.; Ott, H.R. Nuclear Magnetic Resonance and Heavy-Fermion Superconductivity in $(U, T h) B e 13$. Phys. Rev. Lett. 1984, 53, 1833-1836.

31. Shen, Z. X.; Dessau, D. S.; Wells, B. O.; King, D. M.; Spicer, W. E.; Arko, A. J.; Marshall, D.; Lombardo, L. W.; Kapitulnik, A.; Dickinson, P.; Doniach, S.; Dicarlo, J.; Loeser, A. G.; Park C. H. Anomalously Large Gap Anisotropy in the $a-b$ Plane of Bi2Sr2CaCu2O8+. Phys. Rev. Lett. 1993, 70, 1553-1556.

32. Volovik, G. E. Splitting of the Superconducting Transition in High-Temperature Superconductors Due to a Slight Orthorhombic Nature. JETP Lett. 1988, 48, 41-44.

(C) 2010 by the authors; licensee Molecular Diversity Preservation International, Basel, Switzerland. This article is an open-access article distributed under the terms and conditions of the Creative Commons Attribution license (http://creativecommons.org/licenses/by/3.0/). 